\title{
The decompositions with respect to two core non-symmetric cones
}

\author{
Yue Lu · Ching-Yu Yang · Jein-Shan Chen · Hou-Duo Qi
}

Received: date / Accepted: date

\begin{abstract}
It is known that the analysis to tackle with non-symmetric cone optimization is quite different from the way to deal with symmetric cone optimization due to the discrepancy between these types of cones. However, there are still common concepts for both optimization problems, for example, the decomposition with respect to the given cone, smooth and nonsmooth analysis for the associated conic function, conicconvexity, conic-monotonicity and etc. In this paper, motivated by Chares Robert's thesis [Chares, R.: Cones and interior-point algorithms for structured convex optimization involving powers and exponentials. $\mathrm{PhD}$ thesis, UCL-Universite Catholique de Louvain (2009)], we consider the decomposition issue of two core non-symmetric cones, in which two types of decomposition formulae will be proposed, one is adapted from the well-known Moreau decomposition theorem and the other follows from geometry properties of the given cones. As a byproduct, we also establish the conic functions of these cones and generalize the power cone case to its high-dimensional counterpart.
\end{abstract}

Keywords Moreau decomposition theorem · power cone $\cdot$ exponential cone $\cdot$ non-symmetric cones.

Mathematics Subject Classification (2000) 49M27 · 90C25.

\section{Introduction}

Consider the following two core non-symmetric cones

$$
\begin{aligned}
& \mathcal{K}_{\alpha}:=\left\{\left(x_{1}, \bar{x}\right) \in \mathbb{R} \times \mathbb{R}^{2}|| x_{1} \mid \leq \bar{x}_{1}^{\alpha_{1}} \bar{x}_{2}^{\alpha_{2}}, \bar{x}_{1} \geq 0, \bar{x}_{2} \geq 0\right\}, \\
& \mathcal{K}_{\exp }:=\operatorname{cl}\left\{\left(x_{1}, \bar{x}\right) \in \mathbb{R} \times \mathbb{R}^{2} \mid x_{1} \geq \bar{x}_{2} \cdot \exp \left(\frac{\bar{x}_{1}}{\bar{x}_{2}}\right), \bar{x}_{2}>0, x_{1} \geq 0\right\},
\end{aligned}
$$

Yue Lu

School of Mathematical Sciences, Tianjin Normal University, Tianjin 300387, China.

E-mail: jinjin403@sina.com

Ching-Yu Yang

Department of Mathematics, National Taiwan Normal University, Taipei 11677, Taiwan.

E-mail: yangcy@math.ntnu.edu.tw

Jein-Shan Chen $(\varangle)$

Department of Mathematics, National Taiwan Normal University, Taipei 11677, Taiwan.

E-mail: jschen@math.ntnu.edu.tw

Hou-Duo Qi

School of Mathematics, University of Southampton, Southampton SO17 1BJ, United Kingdom.

E-mail: hdqi@soton.ac.uk 
where $\bar{x}:=\left(\bar{x}_{1}, \bar{x}_{2}\right)^{T} \in \mathbb{R}^{2}, \alpha:=\left(\alpha_{1}, \alpha_{2}\right)^{T} \in \mathbb{R}^{2}, \alpha_{1}, \alpha_{2} \in(0,1), \alpha_{1}+\alpha_{2}=1$ and $\operatorname{cl}(\Omega)$ is the closure of $\Omega$. We call $\mathcal{K}_{\alpha}$ the power cone and $\mathcal{K}_{\exp }$ the exponential cone ${ }^{1}$, whose graphs are depicted in Figure 1.
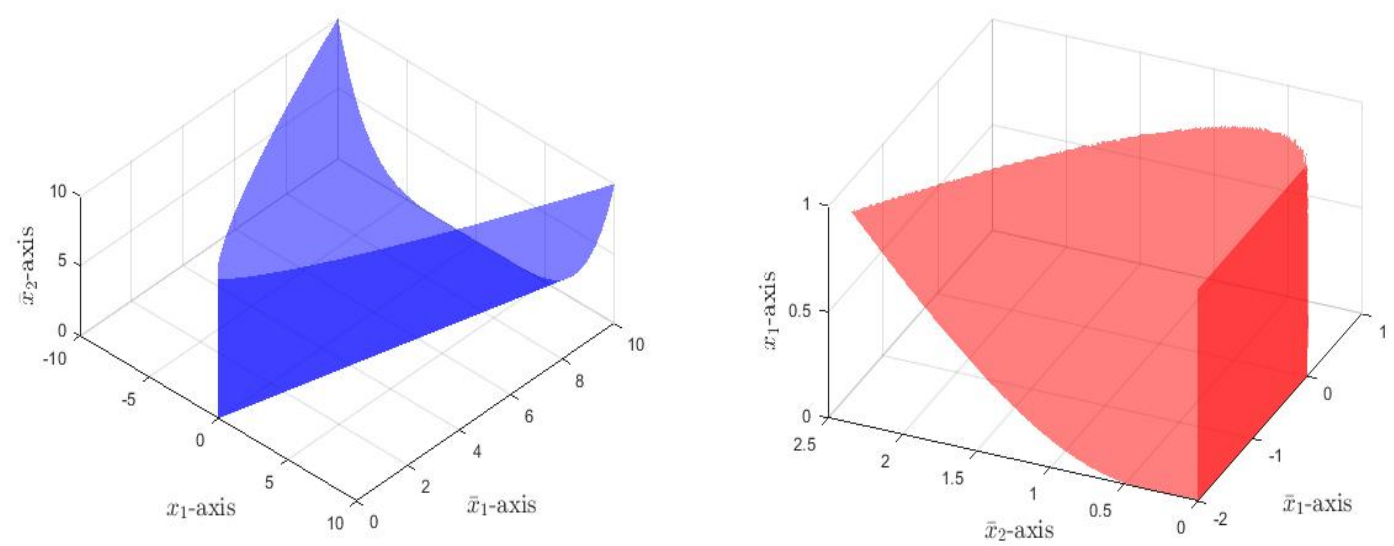

Fig. 1 The power cone $\mathcal{K}_{\alpha}$ (left) and the exponential cone $\mathcal{K}_{\exp }$ (right).

\subsection{Motivations and Literatures}

Why do we pay attention to these two core non-symmetric cones? There are two main reasons. In theory, R. Chares [4] proposes two important concepts (i.e., $\alpha$-representable and extended $\alpha$-representable, see Appendix 6.1) involving powers and exponentials and plenty of famous cones can be generated from these two cones such as second-order cones $[1,22,14,7,9,24]$, $p$-order cones $[2,27,44]$, geometric cones $[3,15,16$, 26], $L_{p}$ cones [17] and etc., one can refer to [4, chapter 4] for more examples. In applications, many practical problems can be cast into optimization models involving the power cone constraints and the exponential cone constraints, such as location problems [4,19] and geometric programming problems [3,31,34] (see Appendix 6.2). Therefore, it becomes quite obvious that there is a great demand for providing systematic studies for these cones.

In the past three decades, a great deal of mathematical effort in conic programming have been devoted to the study of symmetric cones and it has been made extensive progress $[8,13,30,29,33,38]$, particularly for the second-order cone (SOC) $[1,22,14,7,9,24]$ and the positive semi-definite matrix cone (PSD) [41,37, $40,35,39]$. For example, consider the second-order cone

$$
\mathbb{L}^{n}:=\left\{\left(x_{1}, \bar{x}\right) \in \mathbb{R} \times \mathbb{R}^{n-1} \mid x_{1} \geq\|\bar{x}\|\right\} .
$$

For any given $z=\left(z_{1}, \bar{z}\right) \in \mathbb{R} \times \mathbb{R}^{n-1}$, its decomposition with respect to $\mathbb{L}^{n}$ has the form

$$
z=\lambda_{1}(z) \cdot u_{z}^{(1)}+\lambda_{2}(z) \cdot u_{z}^{(2)},
$$

where $\lambda_{i}(z):=z_{1}+(-1)^{i}\|\bar{z}\|$ and $u_{z}^{(i)}$ is equal to $\frac{1}{2}\left(1,(-1)^{i} \frac{\bar{z}}{\|\bar{z}\|}\right)$, if $\bar{z} \neq 0 ; \frac{1}{2}\left(1,(-1)^{i} w\right)$, otherwise, which is applicable for $i=1,2$ with $w \in \mathbb{R}^{n-1}$ being any unit vector. For any scalar function $f: \mathbb{R} \rightarrow \mathbb{R}$, the associated conic function $f^{s o c}(z)$ (called the SOC function) is given by

$$
f^{s o c}(z)=f\left(\lambda_{1}(z)\right) \cdot u_{z}^{(1)}+f\left(\lambda_{2}(z)\right) \cdot u_{z}^{(2)} .
$$

1 The definition of $\mathcal{K}_{\exp }$ used in (2) comes from [4, Section 4.1], which has a slight difference from another form in [34, Definition 2.1.2] as

$$
\mathcal{K}_{\exp }:=\operatorname{cl}\left\{\left(x_{1}, \bar{x}\right) \in \mathbb{R} \times \mathbb{R}^{2} \mid x_{1} \geq \bar{x}_{2} \cdot \exp \left(\frac{\bar{x}_{1}}{\bar{x}_{2}}\right), \bar{x}_{2}>0\right\} .
$$

However, one can observe that these two definitions coincide with each other. 
In light of the decomposition formula and its conic function, one can further establish their analytic properties (i.e., projection mapping, cone-convexity, conic-monotonicity) and design numerical algorithms (i.e., proximal-like algorithms and interior-point algorithms), see Figure 2 for their relations and refer to the monograph [10] for more details. Similar results have also been established for the PSD cone [40,43] and symmetric cones $[38,13]$. Therefore, the past experience $[13,43,10]$ indicates that how to derive the associated decomposition expression with respect to a given cone as the form (3) at a low cost becomes the most important issue in the whole picture of researches.

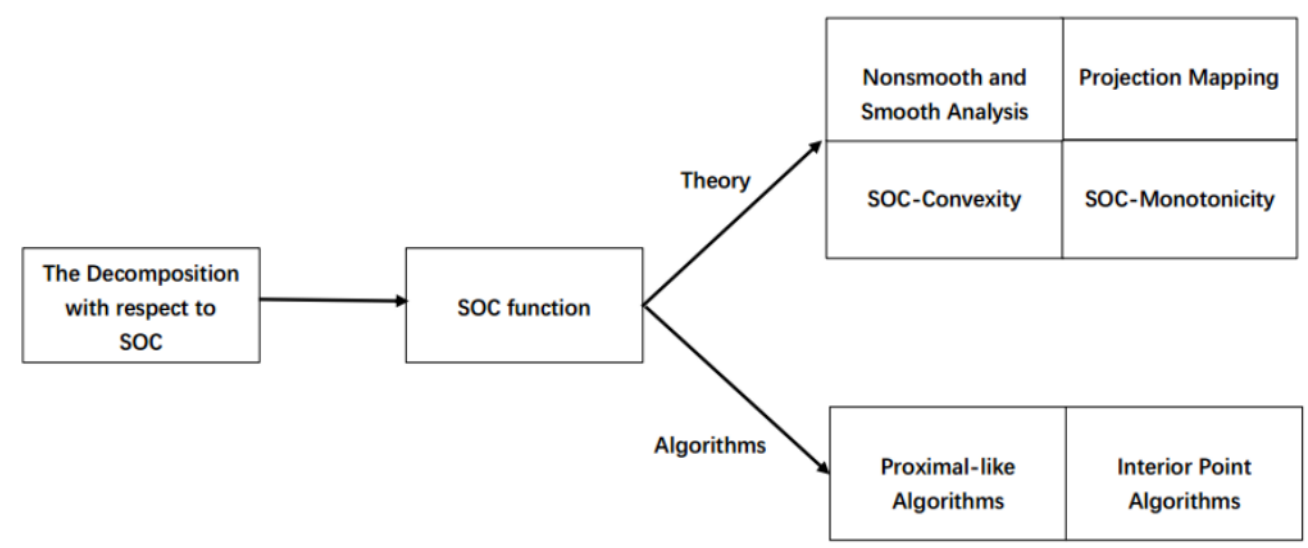

Fig. 2 The relations between the decomposition with respect to SOC and other topics.

As a fundamental tool in optimization, Moreau decomposition theorem [25] characterizes the key relationship between the decomposition with respect to a closed convex cone and its projection mappings. More concretely, for any given $z \in \mathbb{R}^{n}$, it can be uniquely decomposed into

$$
z=\Pi_{\mathcal{K}}(z)+\Pi_{\mathcal{K}^{\circ}}(z)=\Pi_{\mathcal{K}}(z)-\Pi_{\mathcal{K}^{*}}(-z)
$$

where $\Pi_{\mathcal{K}}(z)$ is the projection mapping of $z \in \mathbb{R}^{n}$ onto $\mathcal{K}$ and $\mathcal{K}^{\circ}$ is the polar cone of $\mathcal{K}$, i.e.,

$$
\mathcal{K}^{\circ}:=\left\{y \in \mathbb{R}^{n} \mid x^{T} y \leq 0, \forall x \in \mathcal{K}\right\}
$$

In addition, $\mathcal{K}^{*}$ is the dual cone of $\mathcal{K}$ and satisfies the relation $\mathcal{K}^{*}=-\mathcal{K}^{\circ}$. It follows from (5) that if these projection mappings have closed-form expressions, the decomposition issue can be simply solved by this classical theorem. However, for most non-symmetric cones (except for the circular cone [6, 45], see Appendix $6.3)$, their projection mappings are usually not explicit, such as the power cone $\mathcal{K}_{\alpha}[19$, section 2$]$ and the exponential cone $\mathcal{K}_{\exp }[26$, section 6]. Thus, one cannot employ the Moreau decomposition theorem directly and continue subsequent studies on optimization problems involved with these non-symmetric cones. This is the main and big hurdle for non-symmetric cone optimization problems.

In reality, there are plenty of non-symmetric cones in the literatures, such as homogeneous cones $[5$, $21,42]$, matrix norm cones [11], p-order cones [2,17,44,27], hyperbolicity cones [18,20,32], circular cones $[6,45]$ and copositive cones [12], etc. Unlike the symmetric cone optimization, there seems no systematic study due to the various features and very few algorithms are proposed to solve optimization problems with these non-symmetric cones constraints, except for some interior-point type methods $[44,5,28,36,23]$. For example, Xue and Ye [44] study an optimization problem of minimizing a sum of $p$-norms, in which two new barrier functions are introduced for $p$-order cones and a primal-dual potential reduction algorithm is presented. Chua [5] combines the T-algebra with the primal-dual interior-point algorithm to solve the homogeneous conic programming problems. Based on the concept of self-concordant barriers and the efficient 
computational experience of the long path-following steps, Nesterov [28] proposes a new predictor-corrector path-following method with an additional primal-dual lifting process (called Phase I). Skajaa and Ye [36] present a homogeneous interior-point algorithm for non-symmetric convex conic optimization, in which no Phase I method is needed. Recently, Karimi and Tuncel [23] present a primal-dual interior-point methods for convex optimization problems, in which a new concept called Domain-Driven Setup plays a crucial role in their theoretical analysis.

In contrast to these interior-point type methods, we pay more attention to the decomposition issue of the given cones. It is worth noting that the decompositions with respect to the second-order cone $\mathbb{L}^{n}$ and the circular cone $\mathcal{L}_{\theta}$ (see Eq. (3) and Eq. (51)) show that any given point can be divided into two parts, one lies in the boundary of the given cone (i.e., $u_{z}^{(1)} \in \partial \mathbb{L}^{n}, \tilde{u}_{z}^{(1)} \in \partial \mathcal{L}_{\theta}$, where $\partial \Omega$ is the boundary of $\Omega$ ) and the other comes from the boundary of the given cone (i.e., $u_{z}^{(2)} \in \partial \mathbb{L}^{n}$ ) or its polar (i.e., $\left.\tilde{u}_{z}^{(2)} \in \partial \mathcal{L}_{\theta}^{\circ}\right)$. One can easily verify these results by the Moreau decomposition theorem in some cases (for example, the given point lies out the union of the given cone and its polar), but it is amazing that these decompositions are satisfied in all cases! These observations motivate us to study the boundary structures of the given cones more carefully.

\subsection{Contributions and Contents}

In this paper, we successfully explore two new types of decompositions with respect to the power cone $\mathcal{K}_{\alpha}$ and the exponential cone $\mathcal{K}_{\exp }$, one is adapted from the well-known Moreau decomposition theorem, which looks like

$$
z=s_{x} \cdot x+s_{y} \cdot y, \quad x \in \partial \mathcal{K}, \quad y \in \partial \mathcal{K}^{\circ}, \quad\left(s_{x}, s_{y}\right) \neq(0,0)
$$

and the other follows from geometric structures of the given cone, i.e.,

$$
z=s_{x} \cdot x+s_{y} \cdot y, \quad x \in \partial \mathcal{K}, \quad y \in \partial \mathcal{K}, \quad\left(s_{x}, s_{y}\right) \neq(0,0),
$$

where $z \in \mathbb{R}^{n}, s_{x}, s_{y} \in \mathbb{R}, x, y \in \mathbb{R}^{n}, \mathcal{K}$ has two choices, namely $\mathcal{K}_{\alpha}$ or $\mathcal{K}_{\text {exp }}$, as defined in (1) and (2). In the sequel, we call (6) the Type I decomposition and (7) the Type II decomposition, respectively. To our best knowledge, no results about the decompositions with respect to these two non-symmetric cones have been reported. Hence, the purpose of this paper aims to fill this gap and the contributions of our research can be summarized as follows.

(a) We propose a more compact description of the boundary for these two cones.

(b) Two types of decompositions with respect to $\mathcal{K}_{\alpha}, \mathcal{K}_{\text {exp }}$ are presented, which are do-able and computable. As a byproduct, the decomposition expressions with respect to the high-dimensional power cone are also derived.

(c) We establish the conic functions of the power cone $\mathcal{K}_{\alpha}$ and the exponential cone $\mathcal{K}_{\exp }$ based on their decomposition formulae.

The remainder of this paper is organized as follows. In Sections 2 and 3, we present the decomposition formulae with respect to the power cone $\mathcal{K}_{\alpha}$ and the exponential cone $\mathcal{K}_{\text {exp }}$, respectively. In Section 4 , we discuss some applications of these decompositions. Finally, we draw some concluding remarks in Section 5.

\section{The decompositions with respect to the power cone $\mathcal{K}_{\alpha}$}

In this section, we present two types of decompositions with respect to the power cone $\mathcal{K}_{\alpha}$. Before that, we present some analytic properties of $\mathcal{K}_{\alpha}$ in the following lemmas. 
Lemma $1 \mathcal{K}_{\alpha}$ is a closed convex cone.

Proof It can be easily verified by definition, see Appendix 6.4 for more details.

Lemma 2 The dual cone $\mathcal{K}_{\alpha}^{*}$ can be described as

$$
\mathcal{K}_{\alpha}^{*}=\left\{\left(x_{1}, \bar{x}\right) \in \mathbb{R} \times \mathbb{R}^{2}|| x_{1} \mid \leq\left(\frac{\bar{x}_{1}}{\alpha_{1}}\right)^{\alpha_{1}}\left(\frac{\bar{x}_{2}}{\alpha_{2}}\right)^{\alpha_{2}}, \bar{x}_{1} \geq 0, \bar{x}_{2} \geq 0\right\}
$$

where $\bar{x}:=\left(\bar{x}_{1}, \bar{x}_{2}\right)^{T} \in \mathbb{R}^{2}, \alpha:=\left(\alpha_{1}, \alpha_{2}\right)^{T} \in \mathbb{R}^{2}, \alpha_{1}, \alpha_{2} \in(0,1), \alpha_{1}+\alpha_{2}=1$.

Proof We refer the readers to [4, Theorem 4.3.1] for its verification.

From the relation $\mathcal{K}_{\alpha}^{\circ}=-\mathcal{K}_{\alpha}^{*}$ and Lemma 2 , the polar cone $\mathcal{K}_{\alpha}^{\circ}$ has the following closed-form expression.

Corollary 1 The polar cone $\mathcal{K}_{\alpha}^{\circ}$ is given by

$$
\mathcal{K}_{\alpha}^{\circ}=\left\{\left(x_{1}, \bar{x}\right) \in \mathbb{R} \times \mathbb{R}^{2}|| x_{1} \mid \leq\left(\frac{-\bar{x}_{1}}{\alpha_{1}}\right)^{\alpha_{1}}\left(\frac{-\bar{x}_{2}}{\alpha_{2}}\right)^{\alpha_{2}}, \bar{x}_{1} \leq 0, \bar{x}_{2} \leq 0\right\} .
$$

We now proceed to identify the structures of the power cone $\mathcal{K}_{\alpha}$, its dual $\mathcal{K}_{\alpha}^{*}$ and its polar $\mathcal{K}_{\alpha}^{\circ}$ more clearly, particularly for their interiors and boundaries.

Lemma 3 The interior of the power cone $\mathcal{K}_{\alpha}$, its dual $\mathcal{K}_{\alpha}^{*}$ and its polar $\mathcal{K}_{\alpha}^{\circ}$, denoted by int $\mathcal{K}_{\alpha}$, int $\mathcal{K}_{\alpha}^{*}$ and int $\mathcal{K}_{\alpha}^{\circ}$, are respectively given by

$$
\begin{aligned}
& \operatorname{int}_{\alpha}=\left\{\left(x_{1}, \bar{x}\right) \in \mathbb{R} \times \mathbb{R}^{2}|| x_{1} \mid<\sigma_{\alpha}(\bar{x}), \bar{x}_{1}>0, \bar{x}_{2}>0\right\}, \\
& \text { int } \mathcal{K}_{\alpha}^{*}=\left\{\left(x_{1}, \bar{x}\right) \in \mathbb{R} \times \mathbb{R}^{2}|| x_{1} \mid<\eta_{\alpha}(\bar{x}), \bar{x}_{1}>0, \bar{x}_{2}>0\right\} \text {, } \\
& \operatorname{int}_{\mathcal{\alpha}}^{\circ}=\left\{\left(x_{1}, \bar{x}\right) \in \mathbb{R} \times \mathbb{R}^{2}|| x_{1} \mid<\eta_{\alpha}(-\bar{x}), \bar{x}_{1}<0, \bar{x}_{2}<0\right\},
\end{aligned}
$$

where

$$
\sigma_{\alpha}(\bar{x}):=\bar{x}_{1}^{\alpha_{1}} \bar{x}_{2}^{\alpha_{2}}, \quad \eta_{\alpha}(\bar{x}):=\left(\frac{\bar{x}_{1}}{\alpha_{1}}\right)^{\alpha_{1}}\left(\frac{\bar{x}_{2}}{\alpha_{2}}\right)^{\alpha_{2}}
$$

Proof By definition, $\left(x_{1}, \bar{x}\right)$ is an element of int $\mathcal{K}_{\alpha}$ if and only if there exists an open neighborhood of $\left(x_{1}, \bar{x}\right) \in \mathbb{R} \times \mathbb{R}^{2}$ entirely included in $\mathcal{K}_{\alpha}$. Let us take $\left(x_{1}, \bar{x}\right) \in \mathcal{K}_{\alpha}$. For any given strict positive scalars $\bar{x}_{1}, \bar{x}_{2} \in \mathbb{R}$, it is easy to see that $(0,0,0),\left(0, \bar{x}_{1}, 0\right)$ and $\left(0,0, \bar{x}_{2}\right)$ are all outside of int $\mathcal{K}_{\alpha}$, due to the observation that every open neighborhood with respect to each of these points contains a point with the negative $\bar{x}_{1}$ or $\bar{x}_{2}$ component. For a point $\left(x_{1}, \bar{x}_{1}, \bar{x}_{2}\right) \in \mathbb{R} \times \mathbb{R}^{2}$ such that $\sigma_{\alpha}(\bar{x})=\left|x_{1}\right|$ with $\bar{x}_{1}, \bar{x}_{2}>0$, where $\sigma_{\alpha}(\bar{x})$ is defined as in (11). In this case, we can take a point $\left(x_{1}^{\prime}, \bar{x}_{1}^{\prime}, \bar{x}_{2}^{\prime}\right)$ with $0<\bar{x}_{1}^{\prime}<\bar{x}_{1}, 0<\bar{x}_{2}^{\prime}<\bar{x}_{2}$, $\left|x_{1}^{\prime}\right|>\left|x_{1}\right|$ in every open neighborhood of $\left(x_{1}, \bar{x}_{1}, \bar{x}_{2}\right) \in \mathbb{R} \times \mathbb{R}^{2}$, which implies that $\left|x_{1}^{\prime}\right|>\left|x_{1}\right|=\sigma_{\alpha}(\bar{x})>$ $\sigma_{\alpha}\left(\bar{x}^{\prime}\right)$, i.e., the point $\left(x_{1}^{\prime}, \bar{x}_{1}^{\prime}, \bar{x}_{2}^{\prime}\right)$ can not belong to $\mathcal{K}_{\alpha}$ and hence $\left(x_{1}, \bar{x}_{1}, \bar{x}_{2}\right) \notin \operatorname{int} \mathcal{K}_{\alpha}$.

Next, we turn to show that all the remaining points that do not satisfy the above two cases, i.e., the points in the right-hand side of (8), belong to the interior of $\mathcal{K}_{\alpha}$. For sufficiently small scalar $\epsilon \in\left(0, \min \left\{\bar{x}_{1}, \bar{x}_{2}\right\}\right)$, let $\mathcal{B}_{\left(x_{1}, \bar{x}\right)}^{\epsilon}$ be a neighborhood of $\left(x_{1}, \bar{x}\right)$ with the form

$$
\mathcal{B}_{\left(x_{1}, \bar{x}\right)}^{\epsilon}:=\left\{\left(x_{1}^{\prime}, \bar{x}^{\prime}\right) \in \mathbb{R} \times \mathbb{R}^{2}|0 \leq| x_{1}|-\epsilon \leq| x_{1}^{\prime}|\leq| x_{1} \mid+\epsilon, 0<\bar{x}_{i}-\epsilon \leq \bar{x}_{i}^{\prime} \leq \bar{x}_{i}+\epsilon, i=1,2\right\} .
$$


Taking $\left(x_{1}, \bar{x}\right) \in \mathbb{R} \times \mathbb{R}^{2}$ from the right-hand side of (8), i.e., $\sigma_{\alpha}(\bar{x})>\left|x_{1}\right|, \bar{x}_{i}>0, i=1,2$. For all elements $\left(x_{1}^{\prime}, \bar{x}^{\prime}\right) \in \mathcal{B}_{\left(x_{1}, \bar{x}\right)}^{\epsilon}$, we have

$$
\left|x_{1}^{\prime}\right|-\sigma_{\alpha}\left(\bar{x}^{\prime}\right) \leq\left|\bar{x}_{1}\right|+\epsilon-\left(\bar{x}_{1}^{\prime}\right)^{\alpha_{1}}\left(\bar{x}_{2}^{\prime}\right)^{\alpha_{2}} \leq\left|\bar{x}_{1}\right|+\epsilon-\left(\bar{x}_{1}-\epsilon\right)^{\alpha_{1}}\left(\bar{x}_{2}-\epsilon\right)^{\alpha_{2}} .
$$

In addition, letting $\epsilon \rightarrow 0$, we obtain

$$
\lim _{\epsilon \rightarrow 0}\left(\left|\bar{x}_{1}\right|+\epsilon-\left(\bar{x}_{1}-\epsilon\right)^{\alpha_{1}}\left(\bar{x}_{2}-\epsilon\right)^{\alpha_{2}}\right)=\left|\bar{x}_{1}\right|-\sigma_{\alpha}(\bar{x})<0 .
$$

Therefore, there exists a scalar $\epsilon^{*}$ such that $\left|\bar{x}_{1}\right|+\epsilon^{*}-\left(\bar{x}_{1}-\epsilon^{*}\right)^{\alpha_{1}}\left(\bar{x}_{2}-\epsilon^{*}\right)^{\alpha_{2}}<0$. This together with (12) imply that

$$
\left|x_{1}^{\prime}\right|-\sigma_{\alpha}\left(\bar{x}^{\prime}\right)<0, \forall\left(x_{1}^{\prime}, \bar{x}^{\prime}\right) \in \mathcal{B}_{\left(x_{1}, \bar{x}\right)}^{\epsilon},
$$

which is sufficient to show that $\mathcal{B}_{\left(x_{1}, \bar{x}\right)}^{\epsilon}$ is entirely included in $\mathcal{K}_{\alpha}$ and hence $\left(x_{1}, \bar{x}\right) \in \operatorname{int} \mathcal{K}_{\alpha}$.

Applying a similar way to $\mathcal{K}_{\alpha}^{*}$ and $\mathcal{K}_{\alpha}^{\circ}$, their interiors can also be verified as the right-hand side of (9) and (10).

From the proof of Lemma 3, we further define the following sets

$$
\begin{aligned}
& S_{1}:=\left\{\left(x_{1}, \bar{x}\right) \in \mathbb{R} \times \mathbb{R}^{2} \mid x_{1}=0, \bar{x}_{1}>0, \bar{x}_{2}=0\right\}, \\
& S_{2}:=\left\{\left(x_{1}, \bar{x}\right) \in \mathbb{R} \times \mathbb{R}^{2} \mid x_{1}=0, \bar{x}_{1}=0, \bar{x}_{2}>0\right\}, \\
& S_{3}:=\left\{\left(x_{1}, \bar{x}\right) \in \mathbb{R} \times \mathbb{R}^{2}|| x_{1} \mid=\sigma_{\alpha}(\bar{x}), \bar{x}_{1}>0, \bar{x}_{2}>0\right\}, \\
& S_{4}:=\left\{\left(x_{1}, \bar{x}\right) \in \mathbb{R} \times \mathbb{R}^{2}|| x_{1} \mid=\eta_{\alpha}(\bar{x}), \bar{x}_{1}>0, \bar{x}_{2}>0\right\}, \\
& T_{1}:=\left\{\left(x_{1}, \bar{x}\right) \in \mathbb{R} \times \mathbb{R}^{2} \mid x_{1}=0, \bar{x}_{1}<0, \bar{x}_{2}=0\right\}=-S_{1}, \\
& T_{2}:=\left\{\left(x_{1}, \bar{x}\right) \in \mathbb{R} \times \mathbb{R}^{2} \mid x_{1}=0, \bar{x}_{1}=0, \bar{x}_{2}<0\right\}=-S_{2}, \\
& T_{3}:=\left\{\left(x_{1}, \bar{x}\right) \in \mathbb{R} \times \mathbb{R}^{2}|| x_{1} \mid=\eta_{\alpha}(-\bar{x}), \bar{x}_{1}<0, \bar{x}_{2}<0\right\}=-S_{4} .
\end{aligned}
$$

Then, the boundary of $\mathcal{K}_{\alpha}, \mathcal{K}_{\alpha}^{*}$ and $\mathcal{K}_{\alpha}^{\circ}$ can be stated in a more compact form.

Lemma 4 The boundary of $\mathcal{K}_{\alpha}$ and $\mathcal{K}_{\alpha}^{*}$, denoted by $\partial \mathcal{K}_{\alpha}$ and $\partial \mathcal{K}_{\alpha}^{*}$, are respectively given by

$$
\partial \mathcal{K}_{\alpha}:=S_{1} \cup S_{2} \cup S_{3} \cup\{0\}, \quad \partial \mathcal{K}_{\alpha}^{*}:=S_{1} \cup S_{2} \cup S_{4} \cup\{0\} .
$$

Similarly, the boundary of $\mathcal{K}_{\alpha}^{\circ}$, denoted by $\partial \mathcal{K}_{\alpha}^{\circ}$, can be formulated as

$$
\partial \mathcal{K}_{\alpha}^{\circ}:=T_{1} \cup T_{2} \cup T_{3} \cup\{0\}
$$

Remark 1 It follows that the union set $\mathcal{K}_{\alpha} \cup \mathcal{K}_{\alpha}^{\circ}$ can be divided into seven parts

$$
\mathcal{K}_{\alpha} \cup \mathcal{K}_{\alpha}^{\circ}=S_{1} \cup S_{2} \cup T_{1} \cup T_{2} \cup P_{1} \cup P_{2} \cup\{0\},
$$

where

$$
\begin{aligned}
& P_{1}:=\left\{\left(x_{1}, \bar{x}\right) \in \mathbb{R} \times \mathbb{R}^{2}|| x_{1} \mid \leq \sigma_{\alpha}(\bar{x}), \bar{x}_{1}>0, \bar{x}_{2}>0\right\}, \\
& P_{2}:=\left\{\left(x_{1}, \bar{x}\right) \in \mathbb{R} \times \mathbb{R}^{2}|| x_{1} \mid \leq \eta_{\alpha}(-\bar{x}), \bar{x}_{1}<0, \bar{x}_{2}<0\right\} .
\end{aligned}
$$

In addition, the boundary of $\mathcal{K}_{\alpha}$ and its polar $\mathcal{K}_{\alpha}^{\circ}$ are depicted in Figure 3.

In order to make the classifications clear and neat, we adapt some notations as follows:

$$
z:=\left(z_{1}, \bar{z}\right) \in \mathbb{R} \times \mathbb{R}^{2}, \bar{z}:=\left(\bar{z}_{1}, \bar{z}_{2}\right)^{T} \in \mathbb{R}^{2}, \bar{z}_{\min }:=\min \left\{\bar{z}_{1}, \bar{z}_{2}\right\}, \bar{z}_{\max }:=\max \left\{\bar{z}_{1}, \bar{z}_{2}\right\}
$$

Consequently, we divide the space $\mathbb{R} \times \mathbb{R}^{2}$ into the following four blocks

$$
\begin{array}{ll}
\text { Block I : } & B_{1}:=\left\{\left(z_{1}, \bar{z}\right) \in \mathbb{R} \times \mathbb{R}^{2} \mid \bar{z}_{\min } \cdot \bar{z}_{\max }>0 \text { or }\left(z_{1} \neq 0 \text { and } \bar{z}_{\min }=\bar{z}_{\max }=0\right)\right\} . \\
\text { Block II : } & B_{2}:=\left\{\left(z_{1}, \bar{z}\right) \in \mathbb{R} \times \mathbb{R}^{2} \mid \bar{z}_{\min } \cdot \bar{z}_{\max }=0 \text { and } \bar{z}_{\min }+\bar{z}_{\max } \neq 0\right\} . \\
\text { Block III : } & B_{3}:=\left\{\left(z_{1}, \bar{z}\right) \in \mathbb{R} \times \mathbb{R}^{2} \mid \bar{z}_{\min } \cdot \bar{z}_{\max }<0\right\} . \\
\text { Block IV : } & B_{4}:=\left\{\left(z_{1}, \bar{z}\right) \in \mathbb{R} \times \mathbb{R}^{2} \mid z_{1}=0 \text { and } \bar{z}_{\min }=\bar{z}_{\max }=0\right\} .
\end{array}
$$

The subcases of these blocks with respect to $\mathcal{K}_{\alpha}$ can be found in Table 1. 

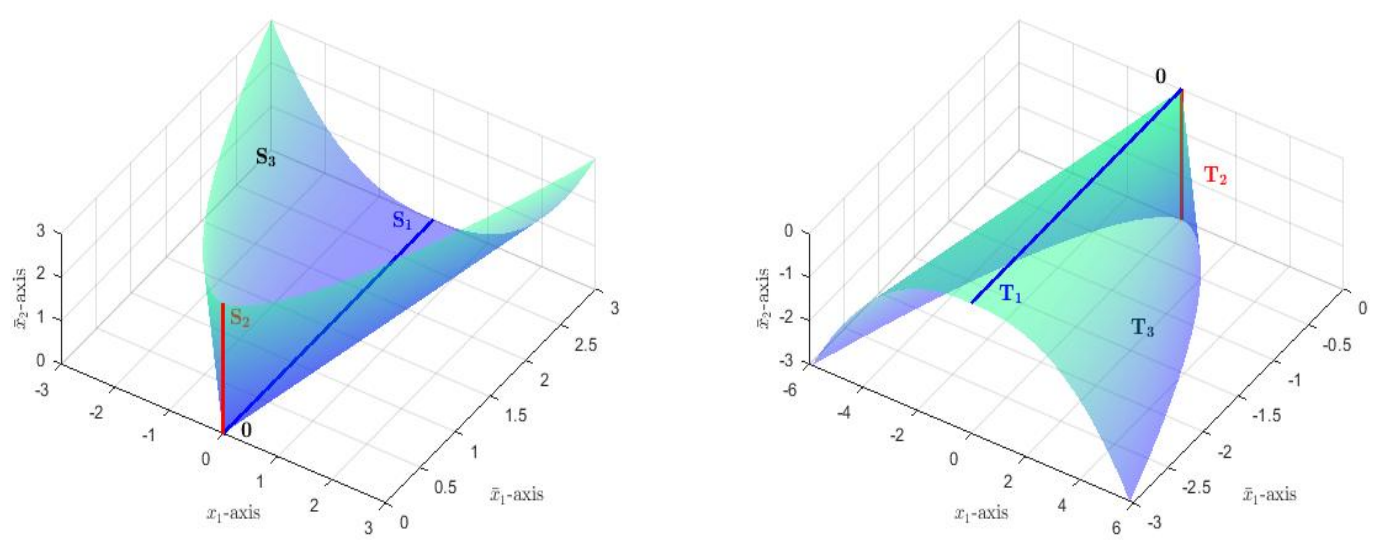

Fig. 3 The different parts of $\partial \mathcal{K}_{\alpha}$ (left) and $\partial \mathcal{K}_{\alpha}^{\circ}$ (right).

Table 1 The subcases of each block in (15) with respect to $\mathcal{K}_{\alpha}$.

\begin{tabular}{cccc}
\hline$B_{1}$ & $B_{2}$ & $B_{3}$ & $B_{4}$ \\
\hline$\left(B_{11}\right) z_{1}$ free, $\bar{z}_{1}>0, \bar{z}_{2}>0$ & $\left(B_{21}\right) z_{1}$ free, $\bar{z}_{1}=0, \bar{z}_{2}>0$ & $\left(B_{31}\right) z_{1}$ free, $\bar{z}_{1}<0, \bar{z}_{2}>0$ & $\left(B_{4}\right) z_{1}=0, \bar{z}_{1}=0, \bar{z}_{2}=0$ \\
$\left(B_{12}\right) z_{1}$ free, $\bar{z}_{1}<0, \bar{z}_{2}<0$ & $\left(B_{22}\right) z_{1}$ free, $\bar{z}_{1}>0, \bar{z}_{2}=0$ & $\left(B_{32}\right) z_{1}$ free, $\bar{z}_{1}>0, \bar{z}_{2}<0$ & \\
$\left(B_{13}\right) z_{1} \neq 0, \bar{z}_{1}=0, \bar{z}_{2}=0$ & $\left(B_{23}\right) z_{1}$ free, $\bar{z}_{1}=0, \bar{z}_{2}<0$ & & \\
& $\left(B_{24}\right) z_{1}$ free, $\bar{z}_{1}<0, \bar{z}_{2}=0$ & & \\
\hline
\end{tabular}

2.1 The Type I decomposition with respect to the power cone $\mathcal{K}_{\alpha}$

In this subsection, we present the Type I decomposition with respect to the power cone $\mathcal{K}_{\alpha}$. To proceed, we discuss four cases, in which the sets $S_{i} \subset \mathcal{K}(i=1,2,3,4)$ and $T_{j} \subset \partial \mathcal{K}^{\circ}(j=1,2,3)$ are defined as in (13).

Case 1: $\left(z_{1}, \bar{z}\right) \in B_{1}$.

(a) $\bar{z}_{\text {min }}>0$. In this subcase, $\left(z_{1}, \bar{z}\right) \in B_{11}$, i.e., $\bar{z}_{1}>0, \bar{z}_{2}>0$, which implies $\sigma_{\alpha}(\bar{z})>0$ and $\eta_{\alpha}(\bar{z})>0$. Then, we take $x=\dot{x}^{\left(B_{1}, a\right)}, y=\dot{y}^{\left(B_{1}, a\right)}$ and $s_{x}=\dot{s}_{x}^{\left(B_{1}, a\right)}, s_{y}=\dot{s}_{y}^{\left(B_{1}, a\right)}$, where

$$
\begin{aligned}
\dot{x}^{\left(B_{1}, a\right)} & :=\left[\begin{array}{c}
1 \\
\frac{\bar{z}}{\sigma_{\alpha}(\bar{z})}
\end{array}\right] \in S_{3}, \dot{y}^{\left(B_{1}, a\right)}:=\left[\begin{array}{c}
1 \\
-\frac{\bar{z}}{\eta_{\alpha}(\bar{z})}
\end{array}\right] \in T_{3} . \\
\dot{s}_{x}^{\left(B_{1}, a\right)} & :=\frac{z_{1}+\eta_{\alpha}(\bar{z})}{\sigma_{\alpha}(\bar{z})+\eta_{\alpha}(\bar{z})} \cdot \sigma_{\alpha}(\bar{z}), \dot{s}_{y}^{\left(B_{1}, a\right)}:=\frac{z_{1}-\sigma_{\alpha}(\bar{z})}{\sigma_{\alpha}(\bar{z})+\eta_{\alpha}(\bar{z})} \cdot \eta_{\alpha}(\bar{z}) .
\end{aligned}
$$

It is easy to show that the above setting satisfies the decomposition formula (6).

(b) $\bar{z}_{\max }<0$. Similar to the argument in Case 1 (a), $\left(z_{1}, \bar{z}\right) \in B_{12}$, i.e., $\bar{z}_{1}<0$, $\bar{z}_{2}<0$, which implies $\sigma_{\alpha}(-\bar{z})>0$ and $\eta_{\alpha}(-\bar{z})>0$. In this subcase, we set $x=\dot{x}^{\left(B_{1}, b\right)}, y=\dot{y}^{\left(B_{1}, b\right)}$ and $s_{x}=\dot{s}_{x}^{\left(B_{1}, b\right)}$, $s_{y}=\dot{s}_{y}^{\left(B_{1}, b\right)}$, where

$$
\begin{aligned}
& \dot{x}^{\left(B_{1}, b\right)}:=\left[\begin{array}{c}
1 \\
\frac{-\bar{z}}{\sigma_{\alpha}(-\bar{z})}
\end{array}\right] \in S_{3}, \dot{y}^{\left(B_{1}, b\right)}:=\left[\begin{array}{c}
1 \\
\frac{\bar{z}}{\eta_{\alpha}(-\bar{z})}
\end{array}\right] \in T_{3} . \\
& \dot{s}_{x}^{\left(B_{1}, b\right)}:=\frac{z_{1}-\eta_{\alpha}(-\bar{z})}{\sigma_{\alpha}(-\bar{z})+\eta_{\alpha}(-\bar{z})} \cdot \sigma_{\alpha}(-\bar{z}), \dot{s}_{y}^{\left(B_{1}, b\right)}:=\frac{z_{1}+\sigma_{\alpha}(-\bar{z})}{\sigma_{\alpha}(-\bar{z})+\eta_{\alpha}(-\bar{z})} \cdot \eta_{\alpha}(-\bar{z}) .
\end{aligned}
$$

(c) $z_{1} \neq 0$ and $\bar{z}_{\text {min }}=\bar{z}_{\max }=0$. In this subcase, $\left(z_{1}, \bar{z}\right) \in B_{13}$, which implies $\sigma_{\alpha}(\bar{z})=0$ and $\eta_{\alpha}(\bar{z})=0$

Therefore, we set $x=\dot{x}^{\left(B_{1}, c\right)}, y=\dot{y}^{\left(B_{1}, c\right)}$ and $s_{x}=\dot{s}_{x}^{\left(B_{1}, c\right)}, s_{y}=\dot{s}_{y}^{\left(B_{1}, c\right)}$, where

$$
\begin{aligned}
& \dot{x}^{\left(B_{1}, c\right)}:=\left[\begin{array}{c}
1 \\
\frac{\mathbf{1}}{\sigma_{\alpha}(\mathbf{1})}
\end{array}\right] \in S_{3}, \dot{y}^{\left(B_{1}, c\right)}:=\left[\begin{array}{c}
1 \\
-\frac{\mathbf{1}}{\eta_{\alpha}(\mathbf{1})}
\end{array}\right] \in T_{3}, \\
& \dot{s}_{x}^{\left(B_{1}, c\right)}:=\frac{z_{1}}{\sigma_{\alpha}(\mathbf{1})+\eta_{\alpha}(\mathbf{1})} \cdot \sigma_{\alpha}(\mathbf{1}), \dot{s}_{y}^{\left(B_{1}, c\right)}:=\frac{z_{1}}{\sigma_{\alpha}(\mathbf{1})+\eta_{\alpha}(\mathbf{1})} \cdot \eta_{\alpha}(\mathbf{1})
\end{aligned}
$$

with $1:=(1,1)^{T} \in \mathbb{R}^{2}$. 
Case 2: $\left(z_{1}, \bar{z}\right) \in B_{2}$.

(a) $\bar{z}_{\min }=0, \bar{z}_{\max }>0$. In this subcase, $\left(z_{1}, \bar{z}\right) \in B_{21}$ or $\left(z_{1}, \bar{z}\right) \in B_{22}$. Therefore, we set $x=\dot{x}^{\left(B_{2}, a\right)}$, $y=\dot{y}^{\left(B_{2}, a\right)}$ and $s_{x}=1, s_{y}=1$, where $\dot{x}^{\left(B_{2}, a\right)}=\left(\dot{x}_{1}^{\left(B_{2}, a\right)}, \dot{\bar{x}}^{\left(B_{2}, a\right)}\right)$ and $\dot{y}^{\left(B_{2}, a\right)}=\left(\dot{y}_{1}^{\left(B_{2}, a\right)}, \dot{\bar{y}}^{\left(B_{2}, a\right)}\right)$ with

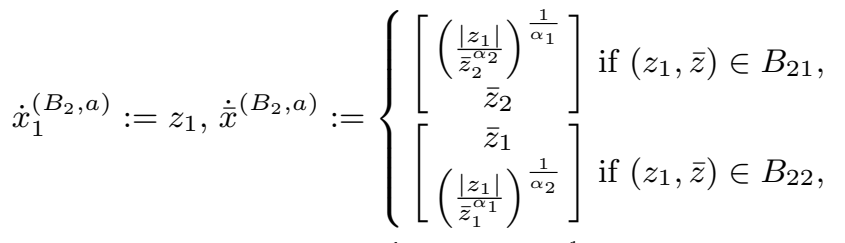

$$
\begin{aligned}
& \dot{y}_{1}^{\left(B_{2}, a\right)}:=0, \dot{\bar{y}}^{\left(B_{2}, a\right)}:=\left\{\begin{array}{l}
{\left[\begin{array}{c}
-\left(\frac{\left|z_{1}\right|}{\bar{z}_{2}^{\alpha_{2}}}\right)^{\frac{1}{\alpha_{1}}} \\
0
\end{array}\right] \text { if }\left(z_{1}, \bar{z}\right) \in B_{21},} \\
{\left[\begin{array}{c}
0 \\
-\left(\frac{\left|z_{1}\right|}{\bar{z}_{1}^{\alpha_{1}}}\right)^{\frac{1}{\alpha_{2}}}
\end{array}\right] \text { if }\left(z_{1}, \bar{z}\right) \in B_{22} .}
\end{array}\right.
\end{aligned}
$$

It is easy to see that
(a) $\left(z_{1}, \bar{z}\right) \in B_{21}, z_{1}=0 \Rightarrow x \in S_{2}, y=0$;
(b) $\left(z_{1}, \bar{z}\right) \in B_{21}, z_{1} \neq 0 \Rightarrow x \in S_{3}, y \in T_{1}$
(c) $\left(z_{1}, \bar{z}\right) \in B_{22}, z_{1}=0 \Rightarrow x \in S_{1}, y=0$;
(d) $\left(z_{1}, \bar{z}\right) \in B_{22}, z_{1} \neq 0 \Rightarrow x \in S_{3}, y \in T_{2}$.

(b) $\bar{z}_{\text {min }}<0, \bar{z}_{\text {max }}=0$. In this subcase, $\left(z_{1}, \bar{z}\right) \in B_{23}$ or $\left(z_{1}, \bar{z}\right) \in B_{24}$. We set $x=\dot{x}^{\left(B_{2}, b\right)}, y=\dot{y}^{\left(B_{2}, b\right)}$ and $s_{x}=-1, s_{y}=-1$, where $\dot{x}^{\left(B_{2}, b\right)}=\left(\dot{x}_{1}^{\left(B_{2}, b\right)}, \dot{\bar{x}}^{\left(B_{2}, b\right)}\right)$ and $\dot{y}^{\left(B_{2}, b\right)}=\left(\dot{y}_{1}^{\left(B_{2}, b\right)}, \dot{\bar{y}}^{\left(B_{2}, b\right)}\right)$ with

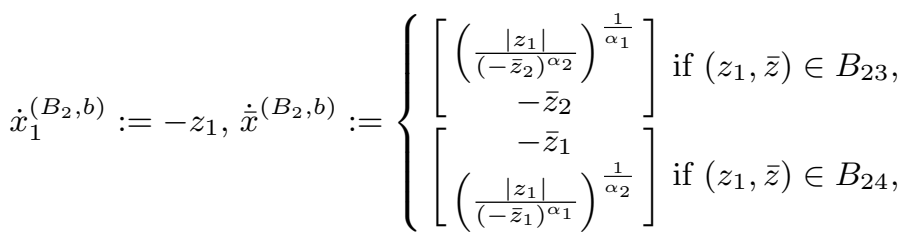

$$
\begin{aligned}
& \dot{y}_{1}^{\left(B_{2}, b\right)}:=0, \quad \dot{\bar{y}}^{\left(B_{2}, b\right)}:=\left\{\begin{array}{l}
{\left[-\left(\frac{\left|z_{1}\right|}{\left(-\bar{z}_{2}\right)^{\alpha_{2}}}\right)^{\frac{1}{\alpha_{1}}}\right] \text { if }\left(z_{1}, \bar{z}\right) \in B_{23},} \\
{\left[\begin{array}{c}
0 \\
-\left(\frac{\left|z_{1}\right|}{\left(-\bar{z}_{1}\right)^{\alpha_{1}}}\right)^{\frac{1}{\alpha_{2}}}
\end{array}\right] \text { if }\left(z_{1}, \bar{z}\right) \in B_{24} .}
\end{array}\right.
\end{aligned}
$$

Similar to the arguments in Case 2 (a), we obtain
(a) $\left(z_{1}, \bar{z}\right) \in B_{23}, z_{1}=0 \Rightarrow x \in S_{2}, y=0$;
(b) $\left(z_{1}, \bar{z}\right) \in B_{23}, z_{1} \neq 0 \Rightarrow x \in S_{3}, y \in T_{1}$;
(c) $\left(z_{1}, \bar{z}\right) \in B_{24}, z_{1}=0 \Rightarrow x \in S_{1}, y=0$;
(d) $\left(z_{1}, \bar{z}\right) \in B_{24}, z_{1} \neq 0 \Rightarrow x \in S_{3}, y \in T_{2}$.

Case 3: $\left(z_{1}, \bar{z}\right) \in B_{3}$. In this subcase, $\left(z_{1}, \bar{z}\right) \in B_{31}$ or $\left(z_{1}, \bar{z}\right) \in B_{32}$. We set $x=\dot{x}^{\left(B_{3}\right)} \in \partial \mathcal{K}_{\alpha}, y=\dot{y}^{\left(B_{3}\right)} \in$ $\partial \mathcal{K}_{\alpha}^{\circ}$ and $s_{x}=1, s_{y}=1$, where $\dot{x}^{\left(B_{3}\right)}=\left(\dot{x}_{1}^{\left(B_{3}\right)}, \dot{\bar{x}}^{\left(B_{3}\right)}\right)$ and $\dot{y}^{\left(B_{3}\right)}=\left(\dot{y}_{1}^{\left(B_{3}\right)}, \dot{\bar{y}}^{\left(B_{3}\right)}\right)$ with

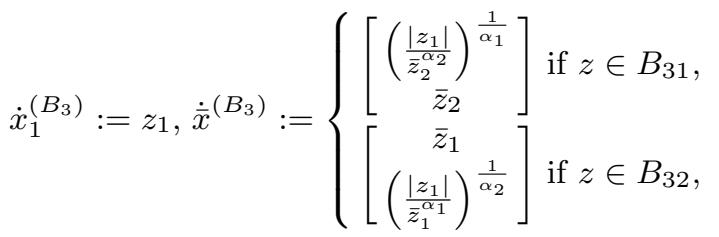

$$
\begin{aligned}
& \dot{y}_{1}^{\left(B_{3}\right)}:=0, \quad \dot{\bar{y}}^{\left(B_{3}\right)}:=\left\{\begin{array}{l}
{\left[\begin{array}{c}
\bar{z}_{1}-\left(\frac{\left|z_{1}\right|}{\bar{z}_{2}^{\alpha_{2}}}\right)^{\frac{1}{\alpha_{1}}} \\
0
\end{array}\right] \text { if } z \in B_{31},} \\
0 \\
{\left[\begin{array}{c}
0 \\
\bar{z}_{2}-\left(\frac{\left|z_{1}\right|}{\bar{z}_{1}^{\alpha_{1}}}\right)^{\frac{1}{\alpha_{2}}}
\end{array}\right] \text { if } z \in B_{32} .}
\end{array}\right.
\end{aligned}
$$

More concretely, we obtain
(a) $\left(z_{1}, \bar{z}\right) \in B_{31}, z_{1}=0 \Rightarrow x \in S_{2}, y \in T_{1}$;
(b) $\left(z_{1}, \bar{z}\right) \in B_{31}, z_{1} \neq 0 \Rightarrow x \in S_{3}, y \in T_{1}$;
(c) $\left(z_{1}, \bar{z}\right) \in B_{32}, z_{1}=0 \Rightarrow x \in S_{1}, y \in T_{2}$;
(d) $\left(z_{1}, \bar{z}\right) \in B_{32}, z_{1} \neq 0 \Rightarrow x \in S_{3}, y \in T_{2}$. 
Case 4: $\left(z_{1}, \bar{z}\right) \in B_{4}$. In this subcase, we set $x=\dot{x}^{\left(B_{4}\right)}, y=\dot{y}^{\left(B_{4}\right)}$ and $s_{x}=1, s_{y}=1$, where

or

$$
\dot{x}^{\left(B_{4}\right)}:=\left[\begin{array}{l}
0 \\
1 \\
0
\end{array}\right] \in S_{1}, \quad \dot{y}^{\left(B_{4}\right)}:=\left[\begin{array}{c}
0 \\
-1 \\
0
\end{array}\right] \in T_{1},
$$

$$
\dot{x}^{\left(B_{4}\right)}:=\left[\begin{array}{l}
0 \\
0 \\
1
\end{array}\right] \in S_{2}, \quad \dot{y}^{\left(B_{4}\right)}:=\left[\begin{array}{c}
0 \\
0 \\
-1
\end{array}\right] \in T_{2} \text {. }
$$

To sum up these discussions, we present the Type I decomposition with respect to the power cone $\mathcal{K}_{\alpha}$ in the following theorem.

Theorem 1 For any given $z=\left(z_{1}, \bar{z}\right) \in \mathbb{R} \times \mathbb{R}^{2}$, its Type I decomposition with respect to $\mathcal{K}_{\alpha}$ is given by

(a) If $z \in B_{1}$, then

$$
z=\left\{\begin{array}{l}
\dot{s}_{x}^{\left(B_{1}, a\right)} \cdot \dot{x}^{\left(B_{1}, a\right)}+\dot{s}_{y}^{\left(B_{1}, a\right)} \cdot \dot{y}^{\left(B_{1}, a\right)}, \text { if } z \in B_{11} \\
\dot{s}_{x}^{\left(B_{1}, b\right)} \cdot \dot{x}^{\left(B_{1}, b\right)}+\dot{s}_{y}^{\left(B_{1}, b\right)} \cdot \dot{y}^{\left(B_{1}, b\right)}, \text { if } z \in B_{12} \\
\dot{s}_{x}^{\left(B_{1}, c\right)} \cdot \dot{x}^{\left(B_{1}, c\right)}+\dot{s}_{y}^{\left(B_{1}, c\right)} \cdot \dot{y}^{\left(B_{1}, c\right)}, \text { if } z \in B_{13}
\end{array}\right.
$$

where $\dot{x}^{\left(B_{1}, a\right)}, \dot{y}^{\left(B_{1}, a\right)}, \dot{s}_{x}^{\left(B_{1}, a\right)}, \dot{s}_{y}^{\left(B_{1}, a\right)}$ are defined as in $(16)-(17), \dot{x}^{\left(B_{1}, b\right)}, \dot{y}^{\left(B_{1}, b\right)}, \dot{s}_{x}^{\left(B_{1}, b\right)}, \dot{s}_{y}^{\left(B_{1}, b\right)}$ are defined as in (18)-(19) and $\dot{x}^{\left(B_{1}, c\right)}, \dot{y}^{\left(B_{1}, c\right)}, \dot{s}_{x}^{\left(B_{1}, c\right)}, \dot{s}_{y}^{\left(B_{1}, c\right)}$ are defined as in (20)-(21).

(b) If $z \in B_{2}$, then

$$
z= \begin{cases}\dot{x}^{\left(B_{2}, a\right)}+\dot{y}^{\left(B_{2}, a\right)}, & \text { if } z \in B_{21} \text { or } z \in B_{22}, \\ (-1) \cdot \dot{x}^{\left(B_{2}, b\right)}+(-1) \cdot \dot{y}^{\left(B_{2}, b\right)}, & \text { if } z \in B_{23} \text { or } z \in B_{24},\end{cases}
$$

where $\dot{x}^{\left(B_{2}, a\right)}, \dot{y}^{\left(B_{2}, a\right)}$ are defined as in (22)-(23), $\dot{x}^{\left(B_{2}, b\right)}, \dot{y}^{\left(B_{2}, b\right)}$ are defined as in (24)-(25).

(c) If $z \in B_{3}$, then $z=\dot{x}^{\left(B_{3}\right)}+\dot{y}^{\left(B_{3}\right)}$, where $\dot{x}^{\left(B_{3}\right)}, \dot{y}^{\left(B_{3}\right)}$ are defined as in (26)-(27).

(d) If $z \in B_{4}$, then $z=\dot{x}^{\left(B_{4}\right)}+\dot{y}^{\left(B_{4}\right)}$, where $\dot{x}^{\left(B_{4}\right)}$ and $\dot{y}^{\left(B_{4}\right)}$ are defined as in (28) or (29).

\begin{tabular}{|c|c|c|c|c|c|c|c|c|}
\hline & \multirow[t]{2}{*}{$\bar{B}_{1}$} & \multicolumn{4}{|c|}{$\bar{B}_{2}$} & \multicolumn{2}{|c|}{$\bar{B}_{3}$} & \multirow[t]{2}{*}{$\bar{B}_{4}$} \\
\hline & & $\bar{B}_{21}$ & $\bar{B}_{22}$ & $\bar{B}_{23}$ & $\bar{B}_{24}$ & $\bar{B}_{31}$ & $\bar{B}_{32}$ & \\
\hline $\begin{array}{l}x_{l o c} \\
y_{l o c}\end{array}$ & $\begin{array}{l}S_{3} \\
T_{3}\end{array}$ & $\begin{array}{l}S_{2} \cup S_{3} \\
\{0\} \cup T_{1}\end{array}$ & $\begin{array}{l}S_{1} \cup S_{3} \\
\{0\} \cup T_{2}\end{array}$ & $\begin{array}{l}S_{2} \cup S_{3} \\
\{0\} \cup T_{1}\end{array}$ & $\begin{array}{l}S_{1} \cup S_{3} \\
\{0\} \cup T_{2}\end{array}$ & $\begin{array}{c}S_{2} \cup S_{3} \\
T_{1}\end{array}$ & $\begin{array}{c}S_{1} \cup S_{3} \\
T_{2}\end{array}$ & $\begin{array}{l}S_{1} \cup S_{2} \\
T_{1} \cup T_{2}\end{array}$ \\
\hline
\end{tabular}

In addition, the locations of the $x$-part and $y$-part in each case are shown in Table 2, where $S_{i}, T_{i}(i=$ $1,2,3,4)$ are defined as in (13) and $x_{l o c}, y_{l o c}$ denote the locations of $x$ and $y$, respectively.

Table 2 The locations of the $x$-part and $y$-part in the Type I decomposition with respect to $\mathcal{K}_{\alpha}$.

2.2 The Type II decomposition with respect to the power cone $\mathcal{K}_{\alpha}$

In this subsection, we present the Type II decomposition with respect to the power cone $\mathcal{K}_{\alpha}$. Similarly, we consider the following four cases.

Case 1: $\left(z_{1}, \bar{z}\right) \in B_{1}$.

(a) $\bar{z}_{\text {min }}>0$. In this subcase, $\left(z_{1}, \bar{z}\right) \in B_{11}$ and $\sigma_{\alpha}(\bar{z})>0$. Then, we take $x=\ddot{x}^{\left(B_{1}, a\right)}, y=\ddot{y}^{\left(B_{1}, a\right)}$ and $s_{x}=\ddot{s}_{x}^{\left(B_{1}, a\right)}, s_{y}=\ddot{s}_{y}^{\left(B_{1}, a\right)}$, where

$$
\begin{aligned}
& \ddot{x}^{\left(B_{1}, a\right)}:=\left[\begin{array}{c}
\frac{1}{\bar{z}} \\
\sigma_{\alpha}(\bar{z})
\end{array}\right] \in S_{3}, \ddot{y}^{\left(B_{1}, a\right)}:=\left[\begin{array}{c}
-1 \\
\frac{\bar{z}}{\sigma_{\alpha}(\bar{z})}
\end{array}\right] \in S_{3} . \\
& \ddot{s}_{x}^{\left(B_{1}, a\right)}:=\frac{z_{1}+\sigma_{\alpha}(\bar{z})}{2}, \ddot{s}_{y}^{\left(B_{1}, a\right)}:=\frac{\sigma_{\alpha}(\bar{z})-z_{1}}{2} .
\end{aligned}
$$


Similarly, we can show that the above setting satisfies the decomposition formula (7).

(b) $\bar{z}_{\max }<0$. Similar to the argument in Case 1 (a), $\left(z_{1}, \bar{z}\right) \in B_{12}$ and $\sigma_{\alpha}(-\bar{z})>0$. In this subcase, we set $x=\ddot{x}^{\left(B_{1}, b\right)}, y=\ddot{y}^{\left(B_{1}, b\right)}$ and $s_{x}=\ddot{s}_{x}^{\left(B_{1}, b\right)}, s_{y}=\ddot{s}_{y}^{\left(B_{1}, b\right)}$, where

$$
\begin{aligned}
& \ddot{x}^{\left(B_{1}, b\right)}:=\left[\begin{array}{c}
1 \\
\frac{-\bar{z}}{\sigma_{\alpha}(-\bar{z})}
\end{array}\right] \in S_{3}, \ddot{y}^{\left(B_{1}, b\right)}:=\left[\begin{array}{c}
-1 \\
\frac{-\bar{z}}{\sigma_{\alpha}(-\bar{z})}
\end{array}\right] \in S_{3} . \\
& \ddot{s}_{x}^{\left(B_{1}, b\right)}:=\frac{z_{1}-\sigma_{\alpha}(-\bar{z})}{2}, \ddot{s}_{y}^{\left(B_{1}, b\right)}:=\frac{-\sigma_{\alpha}(-\bar{z})-z_{1}}{2} .
\end{aligned}
$$

(c) $z_{1} \neq 0$ and $\bar{z}_{\text {min }}=\bar{z}_{\max }=0$. In this subcase, $\left(z_{1}, \bar{z}\right) \in B_{13}$ and $\sigma_{\alpha}(\bar{z})=0$. Thus, we set $x=\ddot{x}^{\left(B_{1}, c\right)}$, $y=\ddot{y}^{\left(B_{1}, c\right)}$ and $s_{x}=\ddot{s}_{x}^{\left(B_{1}, c\right)}, s_{y}=\ddot{s}_{y}^{\left(B_{1}, c\right)}$, where

$$
\begin{aligned}
& \ddot{x}^{\left(B_{1}, c\right)}:=\left[\begin{array}{c}
1 \\
\frac{\mathbf{1}}{\sigma_{\alpha}(\mathbf{1})}
\end{array}\right] \in S_{3}, \ddot{y}^{\left(B_{1}, c\right)}:=\left[\begin{array}{c}
-1 \\
\frac{\mathbf{1}}{\sigma_{\alpha}(\mathbf{1})}
\end{array}\right] \in S_{3}, \\
& \ddot{s}_{x}^{\left(B_{1}, c\right)}=\frac{z_{1}}{2}, \ddot{s}_{y}^{\left(B_{1}, c\right)}=-\frac{z_{1}}{2} .
\end{aligned}
$$

Case 2: $\left(z_{1}, \bar{z}\right) \in B_{2}$.

(a) $\bar{z}_{\text {min }}=0, \bar{z}_{\max }>0$. In this subcase, $\left(z_{1}, \bar{z}\right) \in B_{21}$ or $\left(z_{1}, \bar{z}\right) \in B_{22}$, we set $x=\ddot{x}^{\left(B_{2}, a\right)}, y=\ddot{y}^{\left(B_{2}, a\right)}$ and $s_{x}=1, s_{y}=-1$, where $\ddot{x}^{\left(B_{2}, a\right)}=\left(\ddot{x}_{1}^{\left(B_{2}, a\right)}, \ddot{\bar{x}}^{\left(B_{2}, a\right)}\right)$ and $\ddot{y}^{\left(B_{2}, a\right)}=\left(\ddot{y}_{1}^{\left(B_{2}, a\right)}, \ddot{\bar{y}}^{\left(B_{2}, a\right)}\right)$ with

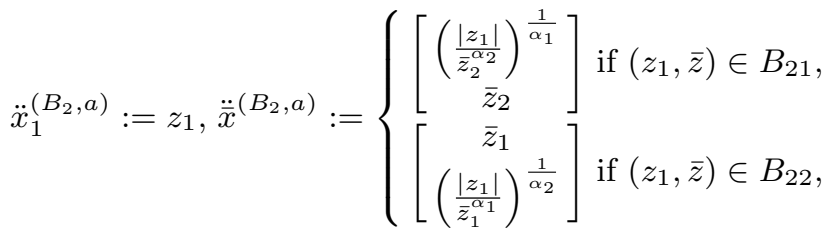

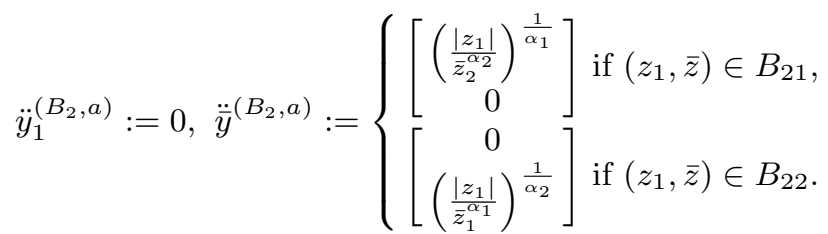

It is easy to see that
(a) $\left(z_{1}, \bar{z}\right) \in B_{21}, z_{1}=0 \Rightarrow x \in S_{2}, y=0$;
(b) $\left(z_{1}, \bar{z}\right) \in B_{21}, z_{1} \neq 0 \Rightarrow x \in S_{3}, y \in S_{1}$;
(c) $\left(z_{1}, \bar{z}\right) \in B_{22}, z_{1}=0 \Rightarrow x \in S_{1}, y=0$;
(d) $\left(z_{1}, \bar{z}\right) \in B_{22}, z_{1} \neq 0 \Rightarrow x \in S_{3}, y \in S_{2}$.

(b) $\bar{z}_{\text {min }}<0, \bar{z}_{\max }=0$. In this subcase, $\left(z_{1}, \bar{z}\right) \in B_{23}$ or $\left(z_{1}, \bar{z}\right) \in B_{24}$. We set $x=\ddot{x}^{\left(B_{2}, b\right)}, y=\ddot{y}^{\left(B_{2}, b\right)}$ and $s_{x}=-1, s_{y}=1$, where $\ddot{x}^{\left(B_{2}, b\right)}=\left(\ddot{x}_{1}^{\left(B_{2}, b\right)}, \ddot{\bar{x}}^{\left(B_{2}, b\right)}\right)$ and $\ddot{y}^{\left(B_{2}, b\right)}=\left(\ddot{y}_{1}^{\left(B_{2}, b\right)}, \ddot{\bar{y}}^{\left(B_{2}, b\right)}\right)$ with

$$
\begin{aligned}
& \ddot{x}_{1}^{\left(B_{2}, b\right)}:=-z_{1}, \ddot{\bar{x}}^{\left(B_{2}, b\right)}:=\left\{\begin{array}{l}
{\left[\left(\frac{\left|z_{1}\right|}{\left(-\bar{z}_{2}\right)^{\alpha_{2}}}\right)^{\frac{1}{\alpha_{1}}}\right] \text { if }\left(z_{1}, \bar{z}\right) \in B_{23},} \\
{\left[\begin{array}{c}
-\bar{z}_{2} \\
\left(\frac{\left|z_{1}\right|}{\left(-\bar{z}_{1}\right)^{\alpha_{1}}}\right)^{\frac{1}{\alpha_{2}}}
\end{array}\right] \text { if }\left(z_{1}, \bar{z}\right) \in B_{24},}
\end{array}\right. \\
& \ddot{y}_{1}^{\left(B_{2}, b\right)}:=0, \quad \ddot{\bar{y}}^{\left(B_{2}, b\right)}:=\left\{\begin{array}{l}
{\left[\left(\frac{\left|z_{1}\right|}{\left(-\bar{z}_{2}\right)^{\alpha_{2}}}\right)^{\frac{1}{\alpha_{1}}}\right] \text { if }\left(z_{1}, \bar{z}\right) \in B_{23},} \\
{\left[\begin{array}{c}
0 \\
\left(\frac{\left|z_{1}\right|}{\left(-\bar{z}_{1}\right)^{\alpha_{1}}}\right)^{\frac{1}{\alpha_{2}}}
\end{array}\right] \text { if }\left(z_{1}, \bar{z}\right) \in B_{24} .}
\end{array}\right.
\end{aligned}
$$

Similar to the arguments in Case 2 (a), we obtain
(a) $\left(z_{1}, \bar{z}\right) \in B_{23}, z_{1}=0 \Rightarrow x \in S_{2}, y=0$;
(b) $\left(z_{1}, \bar{z}\right) \in B_{23}, z_{1} \neq 0 \Rightarrow x \in S_{3}, y \in S_{1}$;
(c) $\left(z_{1}, \bar{z}\right) \in B_{24}, z_{1}=0 \Rightarrow x \in S_{1}, y=0$;
(d) $\left(z_{1}, \bar{z}\right) \in B_{24}, z_{1} \neq 0 \Rightarrow x \in S_{3}, y \in S_{2}$. 
Case 3: $\left(z_{1}, \bar{z}\right) \in B_{3}$. In this subcase, $\left(z_{1}, \bar{z}\right) \in B_{31}$ or $\left(z_{1}, \bar{z}\right) \in B_{32}$. We set $x=\ddot{x}^{\left(B_{3}\right)}, y=\ddot{y}^{\left(B_{3}\right)}$ and $s_{x}=1, s_{y}=-1$, where $\ddot{x}^{\left(B_{3}\right)}=\left(\ddot{x}_{1}^{\left(B_{3}\right)}, \ddot{\bar{x}}^{\left(B_{3}\right)}\right)$ and $\ddot{y}^{\left(B_{3}\right)}=\left(\ddot{y}_{1}^{\left(B_{3}\right)}, \ddot{\bar{y}}^{\left(B_{3}\right)}\right)$ with

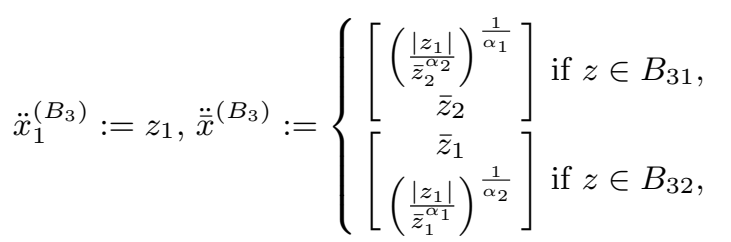

$$
\begin{aligned}
& \ddot{y}_{1}^{\left(B_{3}\right)}:=0, \quad \ddot{\bar{y}}_{j}^{\left(B_{3}\right)}:=\left\{\begin{array}{l}
{\left[\begin{array}{c}
-\bar{z}_{1}+\left(\frac{\left|z_{1}\right|}{\bar{z}_{2}^{\alpha_{2}}}\right)^{\frac{1}{\alpha_{1}}} \\
0
\end{array}\right] \text { if } z \in B_{31},} \\
0 \\
{\left[\begin{array}{c}
0 \\
-\bar{z}_{2}+\left(\frac{\left|z_{1}\right|}{\bar{z}_{1}^{\alpha_{1}}}\right)^{\frac{1}{\alpha_{2}}}
\end{array}\right] \text { if } z \in B_{32} .}
\end{array}\right.
\end{aligned}
$$

More concretely, we obtain
(a) $\left(z_{1}, \bar{z}\right) \in B_{31}, z_{1}=0 \Rightarrow x \in S_{2}, y \in S_{1}$;
(b) $\left(z_{1}, \bar{z}\right) \in B_{31}, z_{1} \neq 0 \Rightarrow x \in S_{3}, y \in S_{1}$;
(c) $\left(z_{1}, \bar{z}\right) \in B_{32}, z_{1}=0 \Rightarrow x \in S_{1}, y \in S_{2}$;
(d) $\left(z_{1}, \bar{z}\right) \in B_{32}, z_{1} \neq 0 \Rightarrow x \in S_{3}, y \in S_{2}$.

Case 4: $\left(z_{1}, \bar{z}\right) \in B_{4}$. In this subcase, we set $x=\ddot{x}^{\left(B_{4}\right)} \in \partial \mathcal{K}_{\alpha}, y=\ddot{y}^{\left(B_{4}\right)} \in \partial \mathcal{K}_{\alpha}$ and $s_{x}=1, s_{y}=-1$, where $\ddot{x}^{\left(B_{4}\right)}=\left(\ddot{x}_{1}^{\left(B_{4}\right)}, \ddot{\bar{x}}^{\left(B_{4}\right)}\right)$ and $\ddot{y}^{\left(B_{4}\right)}=\left(\ddot{y}_{1}^{\left(B_{4}\right)}, \ddot{\bar{y}}^{\left(B_{4}\right)}\right)$ with

$$
\ddot{x}^{\left(B_{4}\right)}:=\left[\begin{array}{l}
0 \\
1 \\
0
\end{array}\right] \in S_{1}, \quad \ddot{y}^{\left(B_{4}\right)}:=\left[\begin{array}{l}
0 \\
1 \\
0
\end{array}\right] \in S_{1},
$$

or

$$
\ddot{x}^{\left(B_{4}\right)}:=\left[\begin{array}{l}
0 \\
0 \\
1
\end{array}\right] \in S_{2}, \quad \ddot{y}^{\left(B_{4}\right)}:=\left[\begin{array}{l}
0 \\
0 \\
1
\end{array}\right] \in S_{2} .
$$

As mentioned above, the next theorem presents the Type II decomposition with respect to the power cone $\mathcal{K}_{\alpha}$.

Theorem 2 For any given $z=\left(z_{1}, \bar{z}\right) \in \mathbb{R} \times \mathbb{R}^{2}$, its Type II decomposition with respect to $\mathcal{K}_{\alpha}$ is given by

(a) If $z \in B_{1}$, then

$$
z=\left\{\begin{array}{l}
\ddot{s}_{x}^{\left(B_{1}, a\right)} \cdot \ddot{x}^{\left(B_{1}, a\right)}+\ddot{s}_{y}^{\left(B_{1}, a\right)} \cdot \ddot{y}^{\left(B_{1}, a\right)}, \text { if } z \in B_{11} \\
\ddot{s}_{x}^{\left(B_{1}, b\right)} \cdot \ddot{x}^{\left(B_{1}, b\right)}+\ddot{s}_{y}^{\left(B_{1}, b\right)} \cdot \ddot{y}^{\left(B_{1}, b\right)}, \quad \text { if } z \in B_{12} \\
\ddot{s}_{x}^{\left(B_{1}, c\right)} \cdot \ddot{x}^{\left(B_{1}, c\right)}+\ddot{s}_{y}^{\left(B_{1}, c\right)} \cdot \ddot{y}^{\left(B_{1}, c\right)}, \quad \text { if } z \in B_{13}
\end{array}\right.
$$

where $\ddot{x}^{\left(B_{1}, a\right)}, \ddot{y}^{\left(B_{1}, a\right)}, \ddot{s}_{x}^{\left(B_{1}, a\right)}, \ddot{s}_{y}^{\left(B_{1}, a\right)}$ are defined as in (30)-(31), $\ddot{x}^{\left(B_{1}, b\right)}, \ddot{y}^{\left(B_{1}, b\right)}, \ddot{s}_{x}^{\left(B_{1}, b\right)}, \ddot{s}_{y}^{\left(B_{1}, b\right)}$ are defined as in (32)-(33) and $\ddot{x}^{\left(B_{1}, c\right)}, \ddot{y}^{\left(B_{1}, c\right)}, \ddot{s}_{x}^{\left(B_{1}, c\right)}, \ddot{s}_{y}^{\left(B_{1}, c\right)}$ are defined as in (34)-(35).

(b) If $z \in B_{2}$, then

$$
z=\left\{\begin{array}{l}
\ddot{x}^{\left(B_{2}, a\right)}+(-1) \cdot \ddot{y}^{\left(B_{2}, a\right)}, \text { if } z \in B_{21} \text { or } z \in B_{22}, \\
(-1) \cdot \ddot{x}^{\left(B_{2}, b\right)}+\ddot{y}^{\left(B_{2}, b\right)}, \text { if } z \in B_{23} \text { or } z \in B_{24},
\end{array}\right.
$$

where $\ddot{x}^{\left(B_{2}, a\right)}, \ddot{y}^{\left(B_{2}, a\right)}$ are defined as (36)-(37), $\ddot{x}^{\left(B_{2}, b\right)}, \ddot{y}^{\left(B_{2}, b\right)}$ are defined as in (38)-(39).

(c) If $z \in B_{3}$, then $z=\ddot{x}^{\left(B_{3}\right)}+(-1) \cdot \ddot{y}^{\left(B_{3}\right)}$, where $\ddot{x}^{\left(B_{3}\right)}$, $\ddot{y}^{\left(B_{3}\right)}$ are defined as in $(40)-(41)$.

(d) If $z \in B_{4}$, then $z=\ddot{x}^{\left(B_{4}\right)}+(-1) \cdot \ddot{y}^{\left(B_{4}\right)}$, where $\ddot{x}^{\left(B_{4}\right)}$ and $\ddot{y}^{\left(B_{4}\right)}$ are defined as in (42) or (43).

In addition, the locations of the $x$-part and $y$-part in each case are summarized in Table 3. 
Table 3 The locations of the $x$-part and $y$-part in the Type II decomposition with respect to $\mathcal{K}_{\alpha}$.

\begin{tabular}{|c|c|c|c|c|c|c|c|c|}
\hline \multirow{2}{*}{\multicolumn{2}{|c|}{$\bar{B}_{1}$}} & \multicolumn{4}{|c|}{$\bar{B}_{2}$} & \multicolumn{2}{|c|}{$\bar{B}_{3}$} & \multirow[t]{2}{*}{$\bar{B}_{4}$} \\
\hline & & $\bar{B}_{21}$ & $\bar{B}_{22}$ & $\bar{B}_{23}$ & $\bar{B}_{24}$ & $\bar{B}_{31}$ & $\bar{B}_{32}$ & \\
\hline $\begin{array}{l}x_{l o c} \\
y_{l o c}\end{array}$ & $\begin{array}{l}S_{3} \\
S_{3}\end{array}$ & $\begin{array}{l}S_{2} \cup S_{3} \\
\{0\} \cup S_{1}\end{array}$ & $\begin{array}{l}S_{1} \cup S_{3} \\
\{0\} \cup S_{2}\end{array}$ & $\begin{array}{c}S_{2} \cup S_{3} \\
\{0\} \cup S_{1}\end{array}$ & $\begin{array}{l}S_{1} \cup S_{3} \\
\{0\} \cup S_{2}\end{array}$ & $\begin{array}{c}S_{2} \cup S_{3} \\
S_{1}\end{array}$ & $\begin{array}{c}S_{1} \cup S_{3} \\
S_{2}\end{array}$ & $\begin{array}{l}S_{1} \cup S_{2} \\
S_{1} \cup S_{2}\end{array}$ \\
\hline
\end{tabular}

\subsection{Manipulation of a real example}

In this subsection, we elaborate more about how to implement the Type I and Type II decomposition with respect to the power cone $\mathcal{K}_{\alpha}$ explicitly by manipulating an example. Without loss of generality, we set the parameters $\alpha_{1}=\alpha_{2}=\frac{1}{2}$.

Example 1 The power cone $\mathcal{K}_{\frac{1}{2}}$ and its polar cone $\mathcal{K}_{\frac{1}{2}}^{\circ}$ are respectively given by

$$
\begin{aligned}
& \mathcal{K}_{\frac{1}{2}}=\left\{\left(x_{1}, \bar{x}\right) \in \mathbb{R} \times \mathbb{R}^{2}|| x_{1} \mid \leq \bar{x}_{1}^{\frac{1}{2}} \bar{x}_{2}^{\frac{1}{2}}, \bar{x}_{1} \geq 0, \bar{x}_{2} \geq 0\right\}, \\
& \mathcal{K}_{\frac{1}{2}}^{\circ}=\left\{\left(x_{1}, \bar{x}\right) \in \mathbb{R} \times \mathbb{R}^{2}|| x_{1} \mid \leq\left(-2 \bar{x}_{1}\right)^{\frac{1}{2}}\left(-2 \bar{x}_{2}\right)^{\frac{1}{2}}, \bar{x}_{1} \leq 0, \bar{x}_{2} \leq 0\right\} .
\end{aligned}
$$

According to the four blocks defined as in (15), we pick different points to figure out their decompositions with respect to $\mathcal{K}_{\frac{1}{2}}$. For example, we take $z=(1,-1,-2)^{T} \in B_{12}$. In this case, $z_{1}=1, \bar{z}=(-1,-2)^{T}$, $\sigma_{\frac{1}{2}}(-\bar{z})=\sqrt{2}, \eta_{\frac{1}{2}}(-\bar{z})=2 \sqrt{2}$. From the relations (18)-(19) and (32)-(33), we obtain

$$
\begin{aligned}
\dot{x}^{\left(B_{1}, b\right)}:=\left[\begin{array}{c}
1 \\
\frac{-\bar{z}}{\sigma_{\frac{1}{2}}(-\bar{z})}
\end{array}\right]=\left[\begin{array}{c}
1 \\
\frac{1}{\sqrt{2}} \\
\frac{2}{\sqrt{2}}
\end{array}\right] \in S_{3}, \quad \dot{y}^{\left(B_{1}, b\right)}:=\left[\begin{array}{c}
1 \\
\frac{\bar{z}}{\eta_{\frac{1}{2}}(-\bar{z})}
\end{array}\right]=\left[\begin{array}{c}
1 \\
-\frac{1}{2 \sqrt{2}} \\
-\frac{1}{\sqrt{2}}
\end{array}\right] \in T_{3}, \\
\dot{s}_{x}^{\left(B_{1}, b\right)}:=\frac{z_{1}-\eta_{\frac{1}{2}}(-\bar{z})}{\sigma_{\frac{1}{2}}(-\bar{z})+\eta_{\frac{1}{2}}(-\bar{z})} \cdot \sigma_{\frac{1}{2}}(-\bar{z})=\frac{1-2 \sqrt{2}}{\sqrt{2}+2 \sqrt{2}} \cdot \sqrt{2}=\frac{1-2 \sqrt{2}}{3}, \\
\dot{s}_{y}^{\left(B_{1}, b\right)}:=\frac{z_{1}+\sigma_{\frac{1}{2}}(-\bar{z})}{\sigma_{\frac{1}{2}}(-\bar{z})+\eta_{\frac{1}{2}}(-\bar{z})} \cdot \eta_{\frac{1}{2}}(-\bar{z})=\frac{1+\sqrt{2}}{\sqrt{2}+2 \sqrt{2}} \cdot 2 \sqrt{2}=\frac{2(1+\sqrt{2})}{3} . \\
\ddot{x}^{\left(B_{1}, b\right)}:=\left[\begin{array}{c}
1 \\
\frac{-\bar{z}}{\sigma_{\frac{1}{2}}(-\bar{z})}
\end{array}\right]=\left[\begin{array}{c}
1 \\
\frac{1}{\sqrt{2}} \\
\frac{2}{\sqrt{2}}
\end{array}\right] \in S_{3}, \quad \ddot{y}^{\left(B_{1}, b\right)}:=\left[\begin{array}{c}
-1 \\
\frac{-\bar{z}}{\sigma_{\frac{1}{2}}(-\bar{z})}
\end{array}\right]=\left[\begin{array}{c}
-1 \\
\frac{1}{\sqrt{2}} \\
\frac{2}{\sqrt{2}}
\end{array}\right] \in S_{3}, \\
\ddot{s}_{x}^{\left(B_{1}, b\right)}:=\frac{z_{1}-\sigma_{\frac{1}{2}}(-\bar{z})}{2}=\frac{1-\sqrt{2}}{2}, \quad \ddot{s}_{y}^{\left(B_{1}, b\right)}:=\frac{-\sigma_{\frac{1}{2}}(-\bar{z})-z_{1}}{2}=\frac{-\sqrt{2}-1}{2} .
\end{aligned}
$$

Therefore, the corresponding two types of decompositions with respect to $\mathcal{K}_{\frac{1}{2}}$ are respectively given by

$$
\begin{aligned}
& \text { Type I: } \quad\left[\begin{array}{c}
1 \\
-1 \\
-2
\end{array}\right]=\frac{1-2 \sqrt{2}}{3}\left[\begin{array}{c}
1 \\
\frac{1}{\sqrt{2}} \\
\frac{2}{\sqrt{2}}
\end{array}\right]+\frac{2(1+\sqrt{2})}{3} \cdot\left[\begin{array}{c}
1 \\
-\frac{1}{2 \sqrt{2}} \\
-\frac{1}{\sqrt{2}}
\end{array}\right], \\
& \text { Type II: } \quad\left[\begin{array}{c}
1 \\
-1 \\
-2
\end{array}\right]=\frac{1-\sqrt{2}}{2}\left[\begin{array}{c}
1 \\
\frac{1}{\sqrt{2}} \\
\frac{2}{\sqrt{2}}
\end{array}\right]+\frac{-\sqrt{2}-1}{2} \cdot\left[\begin{array}{c}
-1 \\
\frac{1}{\sqrt{2}} \\
\frac{2}{\sqrt{2}}
\end{array}\right],
\end{aligned}
$$

whose graphs are depicted in Figure 4 and 5, respectively. The other cases for testing the decompositions with respect to $\mathcal{K}_{\frac{1}{2}}$ can be seen in Table 4 , in which $x_{l o c}, y_{l o c}, z_{l o c}$ denote the locations of $x, y, z$, respectively.

Remark 2 As shown in Example 1, these two types of decompositions for any given nonzero vectors with respect to the power cone $\mathcal{K}_{\alpha}$ are easy to implement, which is a new feature to the progress of this core 


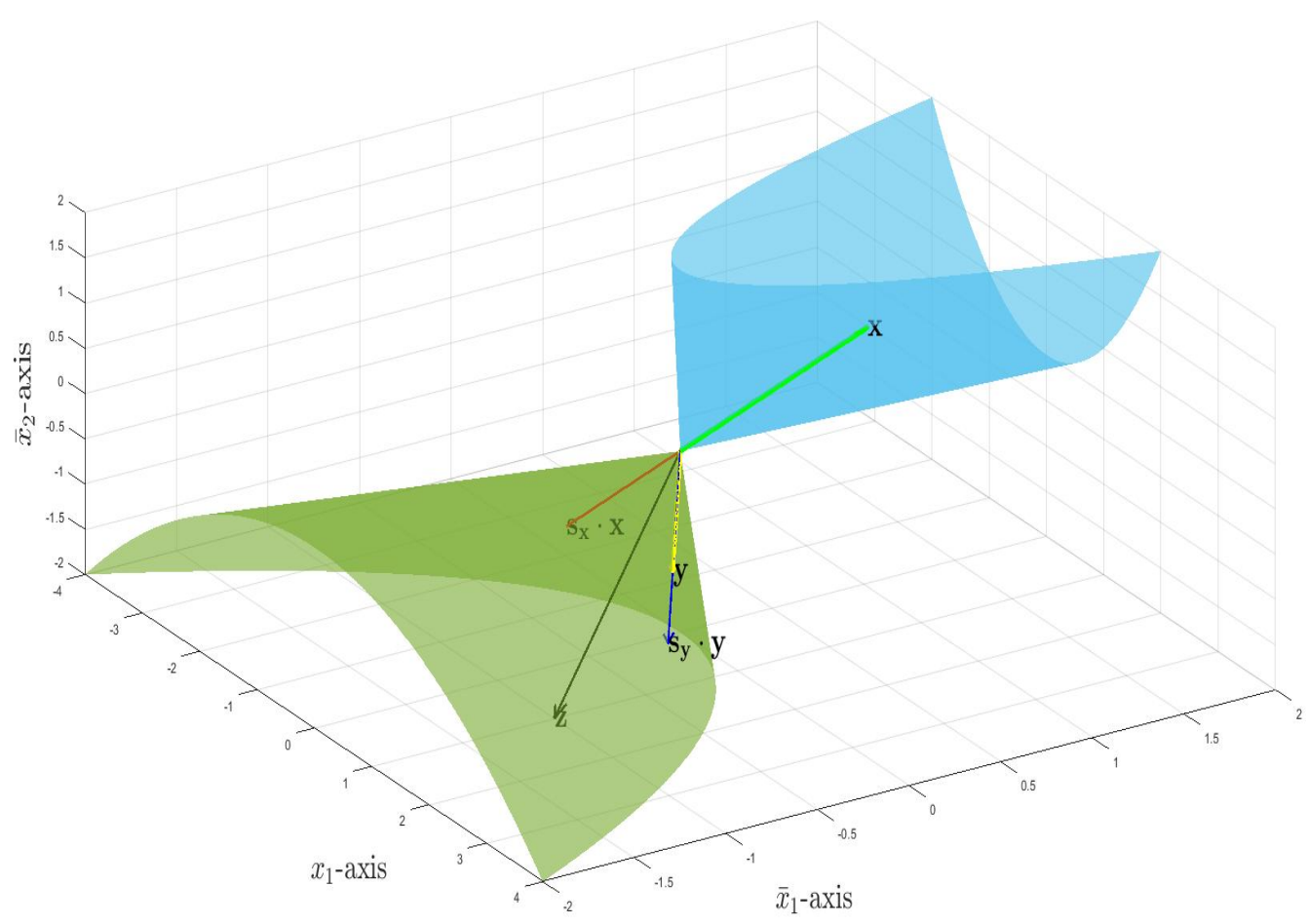

Fig. 4 The Type I decomposition for Example 1.

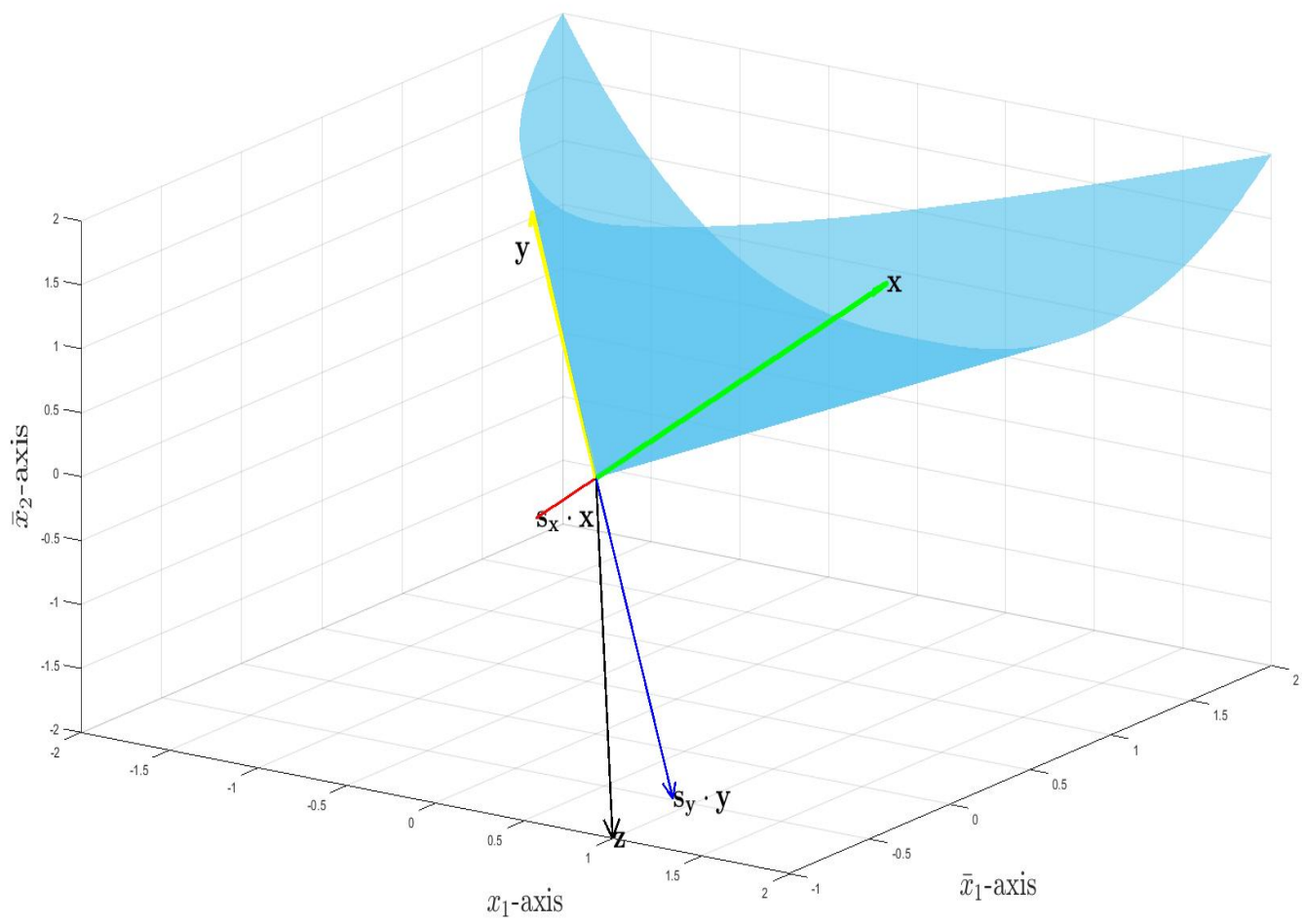

Fig. 5 The Type II decomposition for Example 1. 
Table 4 Examples of two types of decompositions with respect to $\mathcal{K}_{\frac{1}{2}}$.

\begin{tabular}{|c|c|c|c|c|c|c|c|c|c|c|c|c|c|}
\hline \multirow[b]{2}{*}{$z$} & \multirow[b]{2}{*}{$z_{l o c}$} & \multicolumn{6}{|c|}{ Type I } & \multicolumn{6}{|c|}{ Type II } \\
\hline & & $s_{x}$ & $x$ & $x_{l o c}$ & $s_{y}$ & $y$ & $y_{\text {loc }}$ & $s_{x}$ & $x$ & $x_{l o c}$ & $s_{y}$ & $y$ & $y_{\text {loc }}$ \\
\hline$\left[\begin{array}{l}1 \\
2 \\
2\end{array}\right]$ & $B_{1}$ & $\frac{5}{3}$ & {$\left[\begin{array}{l}1 \\
1 \\
1\end{array}\right]$} & $S_{3}$ & $-\frac{2}{3}$ & $\begin{array}{c}1 \\
-\frac{1}{2} \\
-\frac{1}{2}\end{array}$ & $T_{3}$ & $\frac{3}{2}$ & {$\left[\begin{array}{l}1 \\
1 \\
1\end{array}\right]$} & $S_{3}$ & $\frac{1}{2}$ & $\begin{array}{c}-1 \\
1 \\
1\end{array}$ & $S_{3}$ \\
\hline$\left[\begin{array}{l}1 \\
0 \\
1\end{array}\right]$ & $B_{2}$ & 1 & {$\left[\begin{array}{l}1 \\
1 \\
1\end{array}\right]$} & $S_{3}$ & 1 & {$\left[\begin{array}{c}0 \\
-1 \\
0\end{array}\right.$} & $T_{1}$ & 1 & {$\left[\begin{array}{l}1 \\
1 \\
1\end{array}\right]$} & $S_{3}$ & -1 & {$\left[\begin{array}{l}0 \\
1 \\
0\end{array}\right]$} & $S_{1}$ \\
\hline$\left[\begin{array}{c}1 \\
1 \\
-1\end{array}\right]$ & $B_{3}$ & 1 & {$\left[\begin{array}{l}1 \\
1 \\
1\end{array}\right]$} & $S_{3}$ & 1 & {$\left[\begin{array}{c}0 \\
0 \\
-2 \\
\end{array}\right.$} & $T_{2}$ & 1 & {$\left[\begin{array}{l}1 \\
1 \\
1\end{array}\right]$} & $S_{3}$ & -1 & {$\left[\begin{array}{l}0 \\
0 \\
2\end{array}\right]$} & $S_{2}$ \\
\hline$\left[\begin{array}{l}0 \\
0 \\
0\end{array}\right]$ & $B_{4}$ & 1 & $\begin{array}{l}{\left[\begin{array}{l}0 \\
1 \\
0\end{array}\right]} \\
{\left[\begin{array}{l}0 \\
0 \\
1\end{array}\right]}\end{array}$ & $S_{2}$ & 1 & {$\left[\begin{array}{c}0 \\
-1 \\
0\end{array}\right.$} & $T_{2}$ & 1 & {$\left[\begin{array}{l}0 \\
1 \\
0\end{array}\right]$} & $S_{1}$ & -1 & {$\left[\begin{array}{l}0 \\
0 \\
1\end{array}\right]$} & $S_{1}$ \\
\hline
\end{tabular}

non-symmetric cone and plays a crucial role in continuing subsequent study on this topic, for instance generating conic functions like the SOC-function [7] and Löwner's operator for PSD [37,38] as mentioned above. Moreover, through comparing the above two types of decompositions established in Theorem 1 and 2, we rewrite them as follows:

Type I: $\quad z=s_{x}^{I} \cdot x^{I}+s_{y}^{I} \cdot y^{I}$.

Type II: $\quad z=s_{x}^{I I} \cdot x^{I I}+s_{y}^{I I} \cdot y^{I I}$.

It is easy to see that if $\eta_{\alpha}(z)=\sigma_{\alpha}(z)$, then $x^{I}=x^{I I}, y^{I}=-y^{I I}, s_{x}^{I}=s_{x}^{I I}$ and $s_{y}^{I}=-s_{y}^{I I}$, where $\eta_{\alpha}, \sigma_{\alpha}$ are defined as in (11). On the other hand, we also find that the $s_{x}$-part and $s_{y}$-part of the Type I decomposition are more complicated than the Type II counterpart in general. Therefore, we prefer the Type II decomposition with respect to $\mathcal{K}_{\alpha}$ for further studies, see Section 4 for more details.

\section{The decompositions with respect to the exponential cone $\mathcal{K}_{\text {exp }}$}

In this section, we present two types of decompositions with respect to the exponential cone $\mathcal{K}_{\text {exp. Again, }}$ we also present its analytic properties. Due to similar procedures as Section 2, we omit their proofs and only list some results. For the dual of the exponential cone $\mathcal{K}_{\text {exp }}$, we refer the reader to [4, Theorem 4.3.3] for its verification.

Lemma $5 \mathcal{K}_{\exp }$ is a closed convex cone.

Lemma 6 The dual cone $\mathcal{K}_{\exp }^{*}$ can be described as

$$
\mathcal{K}_{\exp }^{*}:=\operatorname{cl}\left\{\left(x_{1}, \bar{x}\right) \in \mathbb{R} \times \mathbb{R}^{2} \mid x_{1} \geq-\frac{\bar{x}_{1}}{e} \cdot \exp \left(\frac{\bar{x}_{2}}{\bar{x}_{1}}\right), \bar{x}_{1}<0, x_{1} \geq 0\right\}
$$

Correspondingly, the polar $\mathcal{K}_{\exp }^{\circ}$ is given by

$$
\mathcal{K}_{\exp }^{\circ}:=\operatorname{cl}\left\{\left(x_{1}, \bar{x}\right) \in \mathbb{R} \times \mathbb{R}^{2} \mid x_{1} \leq-\frac{\bar{x}_{1}}{e} \cdot \exp \left(\frac{\bar{x}_{2}}{\bar{x}_{1}}\right), \bar{x}_{1}>0, x_{1} \leq 0\right\}
$$


Similar to Lemma 4, we also define the following sets

$$
\begin{aligned}
& \hat{S}_{1}:=\left\{\left(x_{1}, \bar{x}\right) \in \mathbb{R} \times \mathbb{R}^{2} \mid x_{1}>0, \bar{x}_{1}<0, \bar{x}_{2}=0\right\}, \\
& \hat{S}_{2}:=\left\{\left(x_{1}, \bar{x}\right) \in \mathbb{R} \times \mathbb{R}^{2} \mid x_{1}=0, \bar{x}_{1}<0, \bar{x}_{2}=0\right\}, \\
& \hat{S}_{3}:=\left\{\left(x_{1}, \bar{x}\right) \in \mathbb{R} \times \mathbb{R}^{2} \mid x_{1}>0, \bar{x}_{1}=0, \bar{x}_{2}=0\right\}, \\
& \hat{S}_{4}:=\left\{\left(x_{1}, \bar{x}\right) \in \mathbb{R} \times \mathbb{R}^{2} \mid x_{1} \geq 0, \sigma_{\exp }(\bar{x})=x_{1}, \bar{x}_{2}>0\right\}, \\
& \hat{S}_{5}:=\left\{\left(x_{1}, \bar{x}\right) \in \mathbb{R} \times \mathbb{R}^{2} \mid x_{1}>0, \bar{x}_{1}=0, \bar{x}_{2}>0\right\}, \\
& \hat{S}_{6}:=\left\{\left(x_{1}, \bar{x}\right) \in \mathbb{R} \times \mathbb{R}^{2} \mid x_{1}=0, \bar{x}_{1}=0, \bar{x}_{2}>0\right\}, \\
& \hat{S}_{7}:=\left\{\left(x_{1}, \bar{x}\right) \in \mathbb{R} \times \mathbb{R}^{2} \mid x_{1}>0, \bar{x}_{1}=0, \bar{x}_{2}=0\right\}, \\
& \hat{S}_{8}:=\left\{\left(x_{1}, \bar{x}\right) \in \mathbb{R} \times \mathbb{R}^{2} \mid x_{1} \geq 0, \bar{x}_{1}<0,-\eta_{\exp }(\bar{x})=x_{1}\right\}, \\
& \hat{T}_{1}:=\left\{\left(x_{1}, \bar{x}\right) \in \mathbb{R} \times \mathbb{R}^{2} \mid x_{1}<0, \bar{x}_{1}=0, \bar{x}_{2}<0\right\}, \\
& \hat{T}_{2}:=\left\{\left(x_{1}, \bar{x}\right) \in \mathbb{R} \times \mathbb{R}^{2} \mid x_{1}=0, \bar{x}_{1}=0, \bar{x}_{2}<0\right\}, \\
& \hat{T}_{3}:=\left\{\left(x_{1}, \bar{x}\right) \in \mathbb{R} \times \mathbb{R}^{2} \mid x_{1}<0, \bar{x}_{1}=0, \bar{x}_{2}=0\right\}, \\
& \hat{T}_{4}:=\left\{\left(x_{1}, \bar{x}\right) \in \mathbb{R} \times \mathbb{R}^{2} \mid x_{1} \leq 0, \bar{x}_{1}>0,-\eta_{\exp }(\bar{x})=x_{1}\right\} .
\end{aligned}
$$

Consequently, the boundary of $\mathcal{K}_{\exp }$ and $\mathcal{K}_{\exp }^{\circ}$ can be described in a more compact form.

Lemma 7 The boundary of $\mathcal{K}_{\exp }$ and $\mathcal{K}_{\exp }^{*}$, denoted by $\partial \mathcal{K}_{\exp }$ and $\partial \mathcal{K}_{\exp }^{*}$, are respectively given by

$$
\partial \mathcal{K}_{\exp }:=\hat{S}_{1} \cup \hat{S}_{2} \cup \hat{S}_{3} \cup \hat{S}_{4} \cup\{0\}, \quad \partial \mathcal{K}_{\exp }^{*}:=\hat{S}_{5} \cup \hat{S}_{6} \cup \hat{S}_{7} \cup \hat{S}_{8} \cup\{0\},
$$

where

$$
\sigma_{\exp }(\bar{x}):=\bar{x}_{2} \cdot \exp \left(\frac{\bar{x}_{1}}{\bar{x}_{2}}\right), \quad \eta_{\exp }(\bar{x}):=\frac{\bar{x}_{1}}{e} \cdot \exp \left(\frac{\bar{x}_{2}}{\bar{x}_{1}}\right) .
$$

Similarly, the boundary of $\mathcal{K}_{\exp }^{\circ}$ can be formulated as

$$
\partial \mathcal{K}_{\exp }^{\circ}:=\hat{T}_{1} \cup \hat{T}_{2} \cup \hat{T}_{3} \cup \hat{T}_{4} \cup\{0\}
$$

Remark 3 Similar to Remark 1, the set $\mathcal{K}_{\exp } \cup \mathcal{K}_{\exp }^{\circ}$ can also be divided into the following nine parts

$$
\mathcal{K}_{\exp } \cup \mathcal{K}_{\exp }^{\circ}=\hat{S}_{1} \cup \hat{S}_{2} \cup \hat{S}_{3} \cup \hat{T}_{1} \cup \hat{T}_{2} \cup \hat{T}_{3} \cup \hat{P}_{1} \cup \hat{P}_{2} \cup\{0\}
$$

where

$$
\begin{aligned}
& \hat{P}_{1}:=\left\{\left(x_{1}, \bar{x}\right) \in \mathbb{R} \times \mathbb{R}^{2} \mid x_{1} \geq 0, \sigma_{\exp }(\bar{x}) \leq x_{1}, \bar{x}_{2}>0\right\}, \\
& \hat{P}_{2}:=\left\{\left(x_{1}, \bar{x}\right) \in \mathbb{R} \times \mathbb{R}^{2} \mid x_{1} \leq 0, \bar{x}_{1}>0,-\eta_{\exp }(\bar{x}) \geq x_{1}\right\} .
\end{aligned}
$$

In addition, the boundary of $\mathcal{K}_{\exp }$ and its polar $\mathcal{K}_{\exp }^{\circ}$ are depicted in Figure 6.
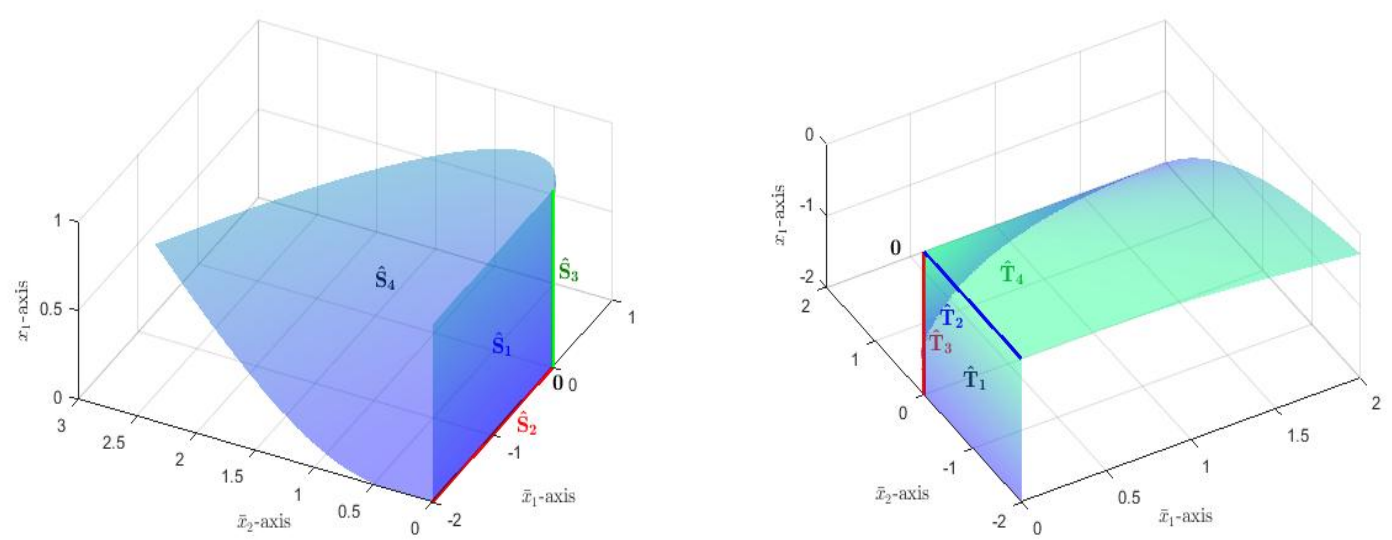

Fig. 6 The different parts of $\partial \mathcal{K}_{\exp }$ (left) and $\partial \mathcal{K}_{\exp }^{\circ}$ (right). 
3.1 The Type I decomposition with respect to the power cone $\mathcal{K}_{\exp }$

In this subsection, we present the Type I decomposition with respect to the exponential cone $\mathcal{K}_{\text {exp }}$, in which we divide the space $\mathbb{R} \times \mathbb{R}^{2}$ into the following four blocks:

Block I : $\quad \tilde{B}_{1}:=\left\{\left(z_{1}, \bar{z}\right) \in \mathbb{R} \times \mathbb{R}^{2} \mid \bar{z}_{1} \cdot \bar{z}_{2}>0\right.$ or $\left(z_{1} \neq 0\right.$ and $\left.\left.\bar{z}=0\right)\right\}$.

Block II : $\quad \tilde{B}_{2}:=\left\{\left(z_{1}, \bar{z}\right) \in \mathbb{R} \times \mathbb{R}^{2} \mid\left(\bar{z}_{1}=0, \bar{z}_{2} \neq 0\right)\right.$ or $\left.\left(\bar{z}_{1}<0, \bar{z}_{2}>0\right)\right\}$.

Block III : $\tilde{B}_{3}:=\left\{\left(z_{1}, \bar{z}\right) \in \mathbb{R} \times \mathbb{R}^{2} \mid\left(\bar{z}_{1} \neq 0, \bar{z}_{2}=0\right)\right.$ or $\left.\left(\bar{z}_{1}>0, \bar{z}_{2}<0\right)\right\}$.

Block IV : $\tilde{B}_{4}:=\left\{\left(z_{1}, \bar{z}\right) \in \mathbb{R} \times \mathbb{R}^{2} \mid z_{1}=0\right.$ and $\left.\bar{z}=0\right\}$.

Their subcases with respect to $\mathcal{K}_{\exp }$ for the Type I decomposition can be found in Table 5 .

Table 5 The subcases of each block in (46) with respect to $\mathcal{K}_{\exp }$ for the Type I decomposition.

\begin{tabular}{cccc}
\hline$\tilde{B}_{1}$ & $\tilde{B}_{2}$ & $\tilde{B}_{3}$ & $\tilde{B}_{4}$ \\
\hline$\left(\tilde{B}_{11}\right) z_{1}$ free, $\bar{z}_{1}>0, \bar{z}_{2}>0$ & $\left(\tilde{B}_{21}\right) z_{1}$ free, $\bar{z}_{1}=0, \bar{z}_{2}>0$ & $\left(\tilde{B}_{31}\right) z_{1}$ free, $\bar{z}_{1}>0, \bar{z}_{2}=0$ & $\left(\tilde{B}_{4}\right) z_{1}=0, \bar{z}_{1}=0, \bar{z}_{2}=0$ \\
$\left(\tilde{B}_{12}\right) z_{1}$ free, $\bar{z}_{1}<0, \bar{z}_{2}<0$ & $\left(\tilde{B}_{22}\right) z_{1}$ free, $\bar{z}_{1}=0, \bar{z}_{2}<0$ & $\left(\tilde{B}_{32}\right) z_{1}$ free, $\bar{z}_{1}<0, \bar{z}_{2}=0$ & \\
$\left(\tilde{B}_{13}\right) z_{1} \neq 0, \bar{z}_{1}=0, \bar{z}_{2}=0$ & $\left(\tilde{B}_{23}\right) z_{1}$ free, $\bar{z}_{1}<0, \bar{z}_{2}>0$ & $\left(\tilde{B}_{33}\right) z_{1}$ free, $\bar{z}_{1}>0, \bar{z}_{2}<0$ & \\
\hline
\end{tabular}

Similar to Theorem 1, we now present the Type I decomposition with respect to $\mathcal{K}_{\exp }$.

Theorem 3 For any given $z=\left(z_{1}, \bar{z}\right) \in \mathbb{R} \times \mathbb{R}^{2}$, its Type I decomposition with respect to $\mathcal{K}_{\exp }$ is given by

(a) If $z \in \tilde{B}_{1}$, then

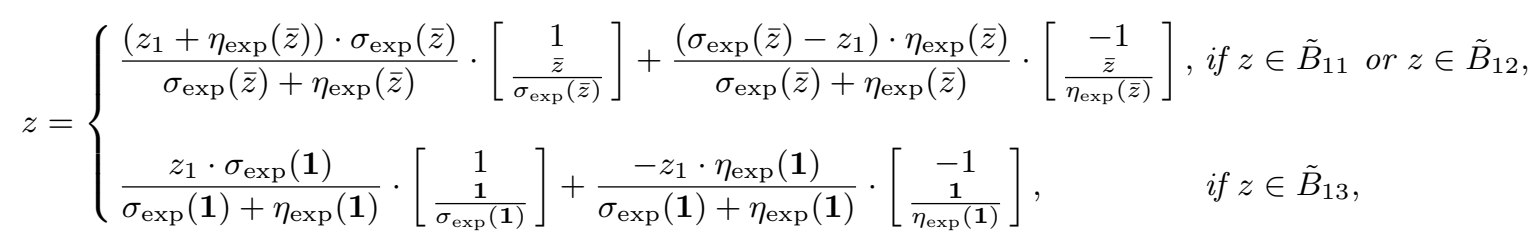

where $\mathbf{1}:=(1,1)^{T} \in \mathbb{R}^{2}$ and $\sigma_{\exp }(\bar{z}), \eta_{\exp }(\bar{z})$ are defined as in $(45)$.

(b) If $z \in \tilde{B}_{2}$, then

$$
z=\sigma_{\exp }(\bar{z}) \cdot\left[\begin{array}{c}
\frac{1}{\bar{z}} \\
\frac{\sigma_{\exp }(\bar{z})}{}
\end{array}\right]+\operatorname{sgn}\left(\sigma_{\exp }(\bar{x})-z_{1}\right) \cdot\left[\begin{array}{c}
-\left|z_{1}-\sigma_{\exp }(\bar{z})\right| \\
0
\end{array}\right],
$$

where $\operatorname{sgn}(t)$ denotes the sign of the variable $t \in \mathbb{R}$.

(c) If $z \in \tilde{B}_{3}$, then

$$
z=\operatorname{sgn}\left(z_{1}+\eta_{\exp }(\bar{z})\right) \cdot\left[\begin{array}{c}
\left|z_{1}+\eta_{\exp }(\bar{z})\right| \\
0
\end{array}\right]+\eta_{\exp }(\bar{z}) \cdot\left[\begin{array}{c}
-1 \\
\frac{\bar{z}}{\eta_{\exp }(\bar{z})}
\end{array}\right]
$$

(d) If $z \in \tilde{B}_{4}$, then

$$
z=1 \cdot\left[\begin{array}{c}
\max \{0, w\} \\
0
\end{array}\right]+1 \cdot\left[\begin{array}{c}
\min \{0,-w\} \\
0
\end{array}\right]
$$

where $w$ is any scalar in $\mathbb{R}$.

In addition, the locations of the $x$-part and $y$-part in each case are summarized in Table 6 , where $\tilde{S}_{i}, \tilde{T}_{i}(i=$ $1,2,3,4)$ are defined as in (44). 
Table 6 The locations of the $x$-part and $y$-part in the Type I decomposition with respect to $\mathcal{K}_{\text {exp }}$

\begin{tabular}{ccccc}
\hline & $\tilde{B}_{1}$ & $\tilde{B}_{2}$ & $\tilde{B}_{3}$ & $\tilde{B}_{4}$ \\
\hline$x_{\text {loc }}$ & $\hat{S}_{4}$ & $\hat{S}_{4}$ & $\{0\} \cup \hat{S}_{3}$ & $\{0\} \cup \hat{S}_{3}$ \\
$y_{l o c}$ & $\hat{T}_{4}$ & $\{0\} \cup \hat{T}_{3}$ & $\hat{T}_{4}$ & $\{0\} \cup \hat{T}_{3}$ \\
\hline
\end{tabular}

3.2 The Type II decomposition with respect to the power cone $\mathcal{K}_{\exp }$

In this subsection, we present the Type II decomposition of the power cone $\mathcal{K}_{\text {exp }}$. By contrast with the Type I case, we present a new space division for $\mathbb{R} \times \mathbb{R}^{2}$ as follows:

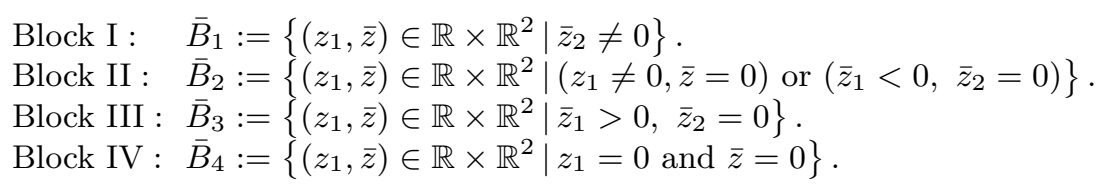

Table 7 indicates their subcases of these blocks with respect to $\mathcal{K}_{\exp }$ for the Type II decomposition.

Table 7 The subcases of each block in (47) with respect to $\mathcal{K}_{\exp }$ for the Type II decomposition.

\begin{tabular}{cccc}
\hline $\bar{B}_{1}$ & $\bar{B}_{2}$ & $\bar{B}_{3}$ & $\bar{B}_{4}$ \\
\hline$\left(\bar{B}_{11}\right) z_{1}$ free, $\bar{z}_{1}>0, \bar{z}_{2}>0$ & $\left(\bar{B}_{21}\right) z_{1} \neq 0, \bar{z}_{1}=0, \bar{z}_{2}=0$ & $\left(\bar{B}_{3}\right) z_{1}$ free, $\bar{z}_{1}>0, \bar{z}_{2}=0$ & $\left(\bar{B}_{4}\right) z_{1}=0, \bar{z}_{1}=0, \bar{z}_{2}=0$ \\
$\left(\bar{B}_{12}\right) z_{1}$ free, $\bar{z}_{1}=0, \bar{z}_{2}>0$ & $\left(\bar{B}_{22}\right) z_{1}$ free, $\bar{z}_{1}<0, \bar{z}_{2}=0$ & & \\
$\left(\bar{B}_{13}\right) z_{1}$ free, $\bar{z}_{1}<0, \bar{z}_{2}>0$ & & & \\
$\left(\bar{B}_{14}\right) z_{1}$ free, $\bar{z}_{1}>0, \bar{z}_{2}<0$ & & & \\
$\left(\bar{B}_{15}\right) z_{1}$ free, $\bar{z}_{1}=0, \bar{z}_{2}<0$ & & & \\
$\left(\bar{B}_{16}\right) z_{1}$ free, $\bar{z}_{1}<0, \bar{z}_{2}<0$ & & & \\
\hline
\end{tabular}

Similar to Theorem 2, the next theorem presents the Type II decomposition with respect to $\mathcal{K}_{\text {exp }}$.

Theorem 4 For any given $z=\left(z_{1}, \bar{z}\right) \in \mathbb{R} \times \mathbb{R}^{2}$, its Type II decomposition with respect to $\mathcal{K}_{\exp }$ is given by

(a) If $z \in \bar{B}_{1}$, then

$$
z=\sigma_{\exp }(\bar{z}) \cdot\left[\begin{array}{c}
1 \\
\frac{\bar{z}}{\sigma_{\exp }(\bar{z})}
\end{array}\right]+\operatorname{sgn}\left(z_{1}-\sigma_{\exp }(\bar{z})\right) \cdot\left[\begin{array}{c}
\left|z_{1}-\sigma_{\exp }(\bar{z})\right| \\
0
\end{array}\right],
$$

where $\operatorname{sgn}(t)$ denotes the sign of the variable $t \in \mathbb{R}$.

(b) If $z \in \bar{B}_{2}$, then

$$
z=1 \cdot\left[\begin{array}{c}
\max \left\{0, z_{1}\right\} \\
\bar{z}
\end{array}\right]+(-1) \cdot\left[\begin{array}{c}
-\min \left\{0, z_{1}\right\} \\
0
\end{array}\right]
$$

(c) If $z \in \bar{B}_{3}$, then

$$
z=1 \cdot\left[\begin{array}{c}
\max \left\{0, z_{1}\right\} \\
0
\end{array}\right]+(-1) \cdot\left[\begin{array}{c}
-\min \left\{0, z_{1}\right\} \\
-\bar{z}
\end{array}\right]
$$

(d) If $z \in \bar{B}_{4}$, then

$$
z=1 \cdot\left[\begin{array}{c}
\max \{0, w\} \\
0
\end{array}\right]+(-1) \cdot\left[\begin{array}{c}
-\min \{0,-w\} \\
0
\end{array}\right]
$$

where $w$ is any scalar in $\mathbb{R}$. 
Table 8 The locations of the $x$-part and $y$-part in the Type II decomposition with respect to $\mathcal{K}_{\exp }$.

\begin{tabular}{|c|c|c|c|c|c|}
\hline & \multirow[t]{2}{*}{$\bar{B}_{1}$} & \multicolumn{2}{|c|}{$\bar{B}_{2}$} & \multirow[t]{2}{*}{$\bar{B}_{3}$} & \multirow[t]{2}{*}{$\bar{B}_{4}$} \\
\hline & & $\bar{B}_{21}$ & $\bar{B}_{22}$ & & \\
\hline $\begin{array}{l}x_{l o c} \\
y_{l o c}\end{array}$ & $\begin{array}{c}\hat{S}_{4} \\
\{0\} \cup \hat{S}_{3}\end{array}$ & $\begin{array}{l}\{0\} \cup \hat{S}_{3} \\
\hat{S}_{3} \cup\{0\}\end{array}$ & $\begin{array}{l}\hat{S}_{1} \cup \hat{S}_{2} \\
\{0\} \cup \hat{S}_{3}\end{array}$ & $\begin{array}{c}\{0\} \cup \hat{S}_{3} \\
\hat{S}_{1} \cup \hat{S}_{2}\end{array}$ & $\begin{array}{l}\{0\} \cup \hat{S}_{3} \\
\{0\} \cup \hat{S}_{3}\end{array}$ \\
\hline
\end{tabular}

In addition, the locations of the $x$-part and $y$-part in each case are summarized in Table 8.

Remark 4 Similar to the power cone $\mathcal{K}_{\alpha}$ case discussed in Section 2.3, Theorem 3 and Theorem 4 also show that our decompositions with respect to the exponential cone $\mathcal{K}_{\exp }$ are easy to calculate. Implementing a real example is routine, we do not repeat it again there. On the other hand, different with the power cone case, the $s_{x}$-part and $s_{y}$-part of the Type I decomposition with respect to $\mathcal{K}_{\exp }$ seems to be more regular than the Type II counterpart in general, due to the appearance of the "wall" part in Fig. 6 (see $\left.\hat{S}_{1} \cup \hat{S}_{2} \cup \hat{S}_{3} \cup\{0\}\right)$. Therefore, we suggest to use the Type I decomposition with respect to $\mathcal{K}_{\exp }$ in the sequential studies.

\section{Applications}

In this section, we discuss some applications of these decompositions with respect to the power cone $\mathcal{K}_{\alpha}$ and the exponential cone $\mathcal{K}_{\text {exp }}$.

\subsection{Conic functions}

As mentioned before, the most important application of the decomposition with respect to the given cone is to establish its associated conic function. In this subsection, we focus on the conic functions for the power cone $\mathcal{K}_{\alpha}$ and the exponential cone $\mathcal{K}_{\text {exp }}$

According to Theorem 2 and Remark 3, the conic function with respect to the power cone $\mathcal{K}_{\alpha}$ is defined in the following form.

Definition 1 For any given $z=\left(z_{1}, \bar{z}\right) \in \mathbb{R} \times \mathbb{R}^{2}$, let $f$ be a scalar function defined in $\mathbb{R}$ and $f^{\text {power }}$ be the conic function with respect to the power cone $\mathcal{K}_{\alpha}$. Denote $\mathbf{1}:=(1,1)^{T} \in \mathbb{R}^{2}$ and the space division of $\mathbb{R} \times \mathbb{R}^{2}$ is defined as in (15). Then, we have

(a) If $z \in B_{1}$, then

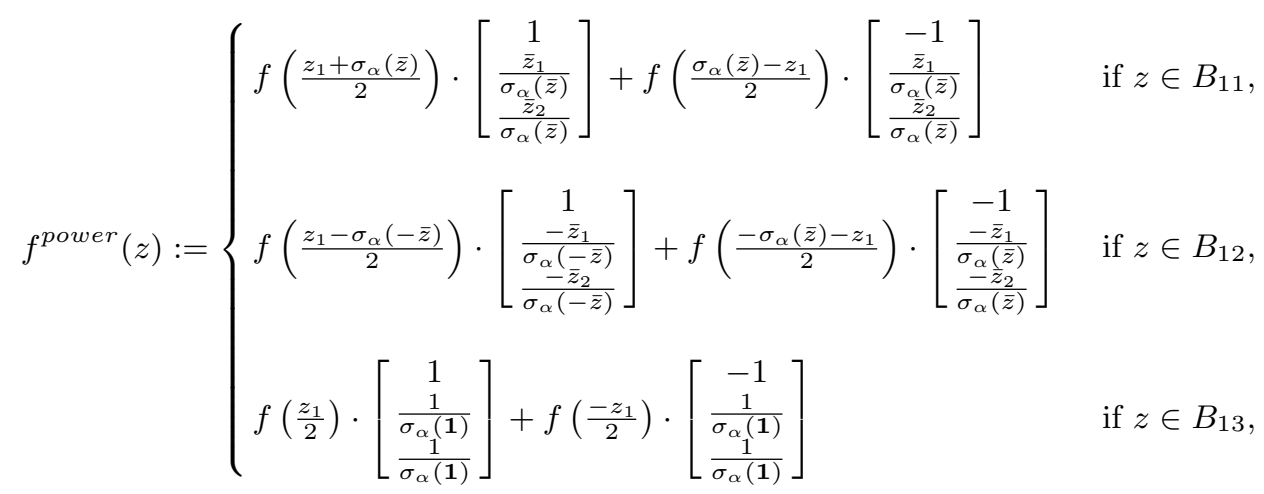


where $\sigma_{\alpha}(\bar{z})$ is defined as in $(11)$.

(b) If $z \in B_{2}$, then

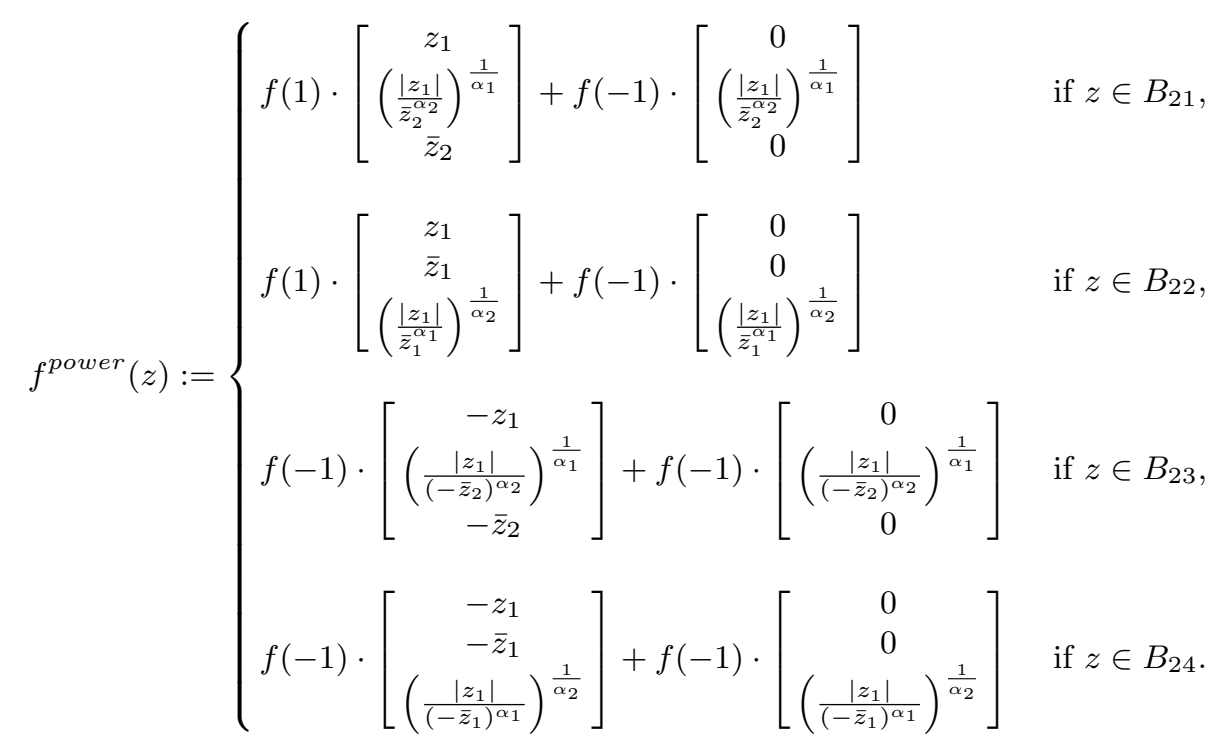

(c) If $z \in B_{3}$, then

$$
f^{\text {power }}(z):=\left\{\begin{array}{c}
f(1) \cdot\left[\begin{array}{c}
z_{1} \\
\left(\frac{\left|z_{1}\right|}{\bar{z}_{2}^{\alpha_{2}}}\right)^{\frac{1}{\alpha_{1}}} \\
\bar{z}_{2}
\end{array}\right]+f(-1) \cdot\left[\begin{array}{c}
0 \\
-\bar{z}_{1}+\left(\frac{\left|z_{1}\right|}{\left.\bar{z}_{2}^{\alpha_{2}}\right)^{\frac{1}{\alpha_{1}}}}\right] \quad \text { if } z \in B_{31}, \\
0
\end{array}\right. \\
f(1) \cdot\left[\begin{array}{c}
z_{1} \\
\bar{z}_{1} \\
\left(\frac{\left|z_{1}\right|}{\bar{z}_{1}^{\alpha_{1}}}\right)^{\frac{1}{\alpha_{2}}}
\end{array}\right]+f(-1) \cdot\left[\begin{array}{c}
0 \\
0 \\
-\bar{z}_{2}+\left(\frac{\left|z_{1}\right|}{\bar{z}_{1}^{\alpha_{1}}}\right)^{\frac{1}{\alpha_{2}}}
\end{array}\right] \quad \text { if } z \in B_{32 .} .
\end{array}\right.
$$

(d) If $z \in B_{4}$, then

$$
f^{\text {power }}(z):=f(1) \cdot\left[\begin{array}{l}
0 \\
1 \\
0
\end{array}\right]+f(-1) \cdot\left[\begin{array}{c}
0 \\
-1 \\
0
\end{array}\right] \quad \text { or } \quad f^{\text {power }}(z):=f(1) \cdot\left[\begin{array}{l}
0 \\
0 \\
1
\end{array}\right]+f(-1) \cdot\left[\begin{array}{c}
0 \\
0 \\
-1
\end{array}\right] \text {. }
$$

Similarly, according to Theorem 3 and Remark 4, the conic function with respect to the exponential cone $\mathcal{K}_{\exp }$ has the following explicit description.

Definition 2 For any given $z=\left(z_{1}, \bar{z}\right) \in \mathbb{R} \times \mathbb{R}^{2}$, let $f$ be a scalar function defined in $\mathbb{R}$ and $f^{\text {exp }}$ be the conic function with respect to the exponential cone $\mathcal{K}_{\text {exp }}$. Denote $\mathbf{1}:=(1,1)^{T} \in \mathbb{R}^{2}$ and the space division of $\mathbb{R} \times \mathbb{R}^{2}$ is defined as in (46). Then, we have

(a) If $z \in \tilde{B}_{1}$, then

$$
f^{\exp }(z):= \begin{cases}f\left(\frac{\left(z_{1}+\eta_{\exp }(\bar{z})\right) \cdot \sigma_{\exp }(\bar{z})}{\sigma_{\exp }(\bar{z})+\eta_{\exp }(\bar{z})}\right) \cdot\left[\frac{1}{\sigma_{\exp }(\bar{z})}\right]+f\left(\frac{\left(\sigma_{\exp }(\bar{z})-z_{1}\right) \cdot \eta_{\exp }(\bar{z})}{\sigma_{\exp }(\bar{z})+\eta_{\exp }(\bar{z})}\right) \cdot\left[\begin{array}{c}
-1 \\
\frac{\bar{z}}{\eta_{\exp }(\bar{z})}
\end{array}\right], \text { if } z \in \tilde{B}_{11} \cup \tilde{B}_{12} \\
f\left(\frac{z_{1} \cdot \sigma_{\exp }(\mathbf{1})}{\sigma_{\exp }(\mathbf{1})+\eta_{\exp }(\mathbf{1})}\right) \cdot\left[\frac{1}{\frac{\mathbf{1}}{\sigma_{\exp }(\mathbf{1})}}\right]+f\left(\frac{-z_{1} \cdot \eta_{\exp }(\mathbf{1})}{\sigma_{\exp }(\mathbf{1})+\eta_{\exp }(\mathbf{1})}\right) \cdot\left[\begin{array}{c}
-1 \\
\left.\frac{\mathbf{1}}{\eta_{\exp }(\mathbf{1})}\right],
\end{array}\right.\end{cases}
$$

where $\sigma_{\exp }(\bar{z}), \eta_{\exp }(\bar{z})$ are defined as in (45). 
(b) If $z \in \tilde{B}_{2}$, then

$$
f^{\exp }(z):=f\left(\sigma_{\exp }(\bar{z})\right) \cdot\left[\begin{array}{c}
1 \\
\frac{\bar{z}}{\sigma_{\exp }(\bar{z})}
\end{array}\right]+f\left(\operatorname{sgn}\left(\sigma_{\exp }(\bar{x})-z_{1}\right)\right) \cdot\left[\begin{array}{c}
-\left|z_{1}-\sigma_{\exp }(\bar{z})\right| \\
0
\end{array}\right],
$$

where $\operatorname{sgn}(t)$ denotes the sign of the variable $t \in \mathbb{R}$.

(c) If $z \in \tilde{B}_{3}$, then

$$
f^{\exp }(z):=f\left(\operatorname{sgn}\left(z_{1}+\eta_{\exp }(\bar{z})\right)\right) \cdot\left[\begin{array}{c}
\left|z_{1}+\eta_{\exp }(\bar{z})\right| \\
0
\end{array}\right]+f\left(\eta_{\exp }(\bar{z})\right) \cdot\left[\begin{array}{c}
-1 \\
\bar{z} \\
\eta_{\exp }(\bar{z})
\end{array}\right] .
$$

(d) If $z \in \tilde{B}_{4}$, then

$$
f^{e x p}(z):=f(1) \cdot\left[\begin{array}{c}
\max \{0, w\} \\
0
\end{array}\right]+f(1) \cdot\left[\begin{array}{c}
\min \{0,-w\} \\
0
\end{array}\right],
$$

where $w$ is any scalar in $\mathbb{R}$.

4.2 The generalization to the high-dimensional power cone

In this subsection, we extend the discussion for the power cone $\mathcal{K}_{\alpha}$ to its high-dimensional version

$$
\mathcal{K}_{\alpha}^{(n)}:=\left\{\left(x_{1}, \bar{x}\right) \in \mathbb{R} \times \mathbb{R}^{n}|| x_{1} \mid \leq \prod_{i=1}^{n} \bar{x}_{i}^{\alpha_{i}}, \bar{x}_{i} \geq 0, i=1,2, \cdots, n\right\},
$$

where $\bar{x}:=\left(\bar{x}_{1}, \bar{x}_{2}, \cdots, \bar{x}_{n}\right)^{T} \in \mathbb{R}^{n}$. In order to make the classifications clear and neat, we similarly adapt some notations as follows:

$$
\begin{gathered}
\bar{z}:=\left(\bar{z}_{1}, \bar{z}_{2}, \cdots, \bar{z}_{n}\right)^{T} \in \mathbb{R}^{n}, \bar{z}_{\min }:=\min \left\{\bar{z}_{1}, \bar{z}_{2}, \cdots, \bar{z}_{n}\right\}, \bar{z}_{\max }:=\max \left\{\bar{z}_{1}, \bar{z}_{2}, \cdots, \bar{z}_{n}\right\}, \\
\mathbf{1}^{(n)}:=(1,1, \cdots, 1)^{T} \in \mathbb{R}^{n}, \mathbf{1}_{k}:=(0, \cdots, 1, \cdots, 0)^{T} \in \mathbb{R}^{n},[\mathbf{n}]:=\{1,2, \cdots, n\}, \\
I_{-}:=\left\{i \in[\mathbf{n}] \mid \bar{z}_{i}<0\right\}, I_{0}:=\left\{i \in[\mathbf{n}] \mid \bar{z}_{i}=0\right\}, I_{+}:=\left\{i \in[\mathbf{n}] \mid \bar{z}_{i}>0\right\}, \\
\sigma_{\alpha}^{(n)}(\bar{z}):=\prod_{i=1}^{n} \bar{z}_{i}^{\alpha_{i}}, \quad \eta_{\alpha}^{(n)}(\bar{z}):=\prod_{i=1}^{n}\left(\frac{\bar{z}_{i}}{\alpha_{i}}\right)^{\alpha_{i}}
\end{gathered}
$$

where $\mathbf{1}_{k}(k=1,2, \cdots, n)$ is the $k$ th column of the identity matrix $I_{n} \in \mathbb{R}^{n \times n}$. Now, the space $\mathbb{R} \times \mathbb{R}^{n}$ can be divided into the following four blocks

Block I : $\quad B_{1}^{(n)}:=\left\{\left(z_{1}, \bar{z}\right) \in \mathbb{R} \times \mathbb{R}^{n} \mid \bar{z}_{\min } \cdot \bar{z}_{\max }>0\right.$ or $\left(\bar{z}_{\min }=\bar{z}_{\max }=0\right.$ and $\left.\left.z_{1} \neq 0\right)\right\}$.

Block II : $\quad B_{2}^{(n)}:=\left\{\left(z_{1}, \bar{z}\right) \in \mathbb{R} \times \mathbb{R}^{n} \mid \bar{z}_{\min } \cdot \bar{z}_{\max }=0\right.$ and $\left.\bar{z}_{\min }+\bar{z}_{\max } \neq 0\right\}$.

Block III : $B_{3}^{(n)}:=\left\{\left(z_{1}, \bar{z}\right) \in \mathbb{R} \times \mathbb{R}^{n} \mid \bar{z}_{\min } \cdot \bar{z}_{\max }<0\right\}$.

Block IV : $B_{4}^{(n)}:=\left\{\left(z_{1}, \bar{z}\right) \in \mathbb{R} \times \mathbb{R}^{n} \mid \bar{z}_{\min }=\bar{z}_{\max }=0\right.$ and $\left.z_{1}=0\right\}$.

We now establish two types of decompositions with respect to $\mathcal{K}_{\alpha}^{(n)}$ defined as in (48) in the following theorems. The proofs are adapted from Theorem 1 and 2, we omit their details and only list the results.

Theorem 5 For any given $z=\left(z_{1}, \bar{z}\right) \in \mathbb{R} \times \mathbb{R}^{n}$, its Type I decomposition with respect to $\mathcal{K}_{\alpha}^{(n)}$ is given by

(a) If $z \in B_{1}^{(n)}$, then

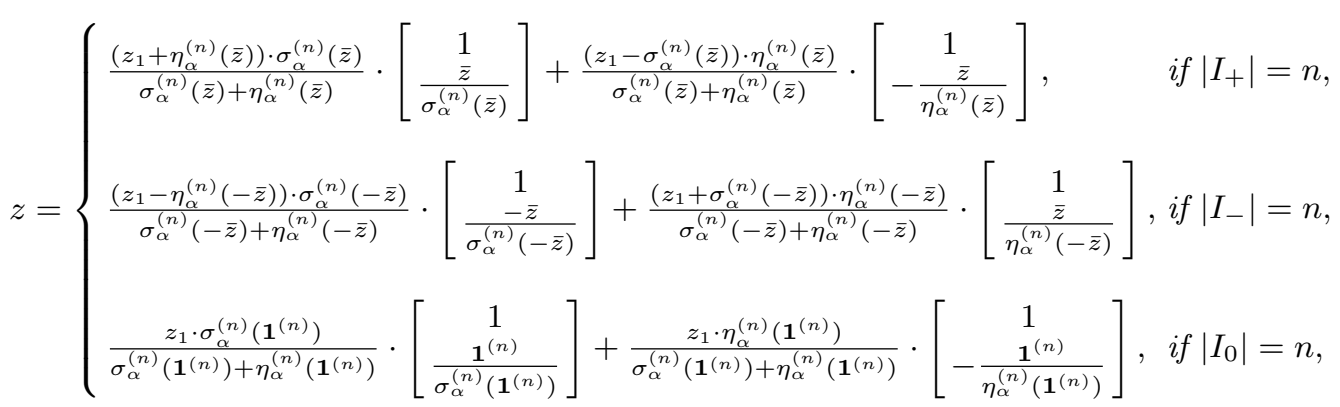


where $\mathbf{1}^{(n)}, \sigma_{\alpha}^{(n)}(\bar{x}), \eta_{\alpha}^{(n)}(\bar{x})$ are defined as in (49) and $|I|$ denotes the cardinality of $I$.

(b) If $z \in B_{2}^{(n)}$, then

$$
z= \begin{cases}1 \cdot\left[\begin{array}{c}
z_{1} \\
\dot{\bar{x}}^{\left(B_{2}^{(n)}, a\right)}
\end{array}\right]+1 \cdot\left[\begin{array}{c}
0 \\
\dot{\bar{y}}^{\left(B_{2}^{(n)}, a\right)}
\end{array}\right], & \text { if }\left|I_{-}\right|=0, \\
(-1) \cdot\left[\begin{array}{c}
-z_{1} \\
\dot{\bar{x}}^{\left(B_{2}^{(n)}, b\right)}
\end{array}\right]+(-1) \cdot\left[\begin{array}{c}
0 \\
\dot{\bar{y}}^{\left(B_{2}^{(n)}, b\right)}
\end{array}\right], \text { if }\left|I_{+}\right|=0,\end{cases}
$$

where $k$ is the smallest index in $I_{0}$ and $\dot{\bar{x}}^{\left(B_{2}^{(n)}, a\right)}, \dot{\bar{y}}^{\left(B_{2}^{(n)}, a\right)}, \dot{\bar{x}}_{2}^{\left(B_{2}^{(n)}, b\right)}, \dot{\bar{y}}^{\left(B_{2}^{(n)}, b\right)}$ are respectively defined as follows:

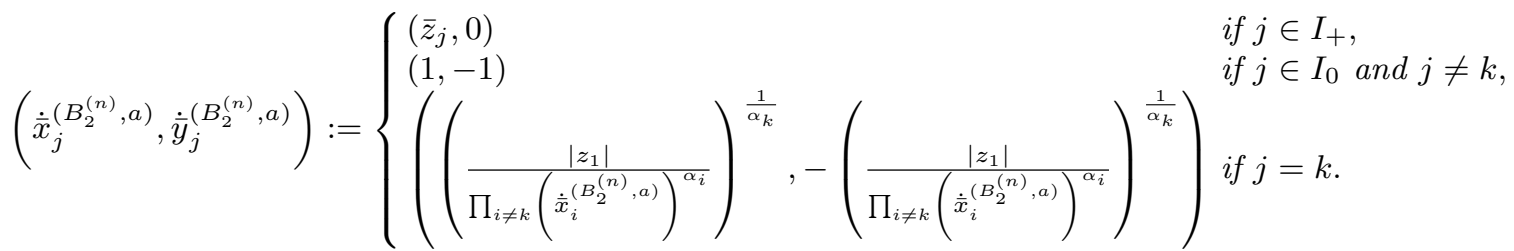

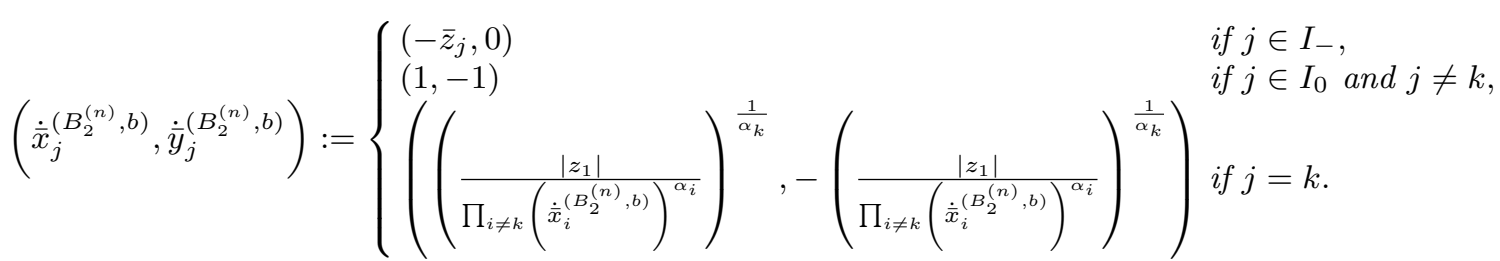

(c) If $z \in B_{3}^{(n)}$, then

$$
z=\left\{\begin{array}{l}
1 \cdot\left[\begin{array}{c}
z_{1} \\
\dot{\bar{x}}^{\left(B_{3}^{(n)}, a\right)}
\end{array}\right]+1 \cdot\left[\begin{array}{c}
0 \\
\dot{\bar{y}}^{\left(B_{3}^{(n)}, a\right)}
\end{array}\right], \text { if }\left|I_{0}\right|=0, \\
1 \cdot\left[\begin{array}{c}
z_{1} \\
\dot{\bar{x}}^{\left(B_{3}^{(n)}, b\right)}
\end{array}\right]+1 \cdot\left[\begin{array}{c}
0 \\
\dot{\bar{y}}^{\left(B_{3}^{(n)}, b\right)}
\end{array}\right], \text { if }\left|I_{0}\right| \neq 0,
\end{array}\right.
$$

where $t$ is the smallest index in $I_{-}$and $\dot{\bar{x}}^{\left(B_{3}^{(n)}, a\right)}, \dot{\bar{y}}^{\left(B_{3}^{(n)}, a\right)}$ are respectively defined as follows:

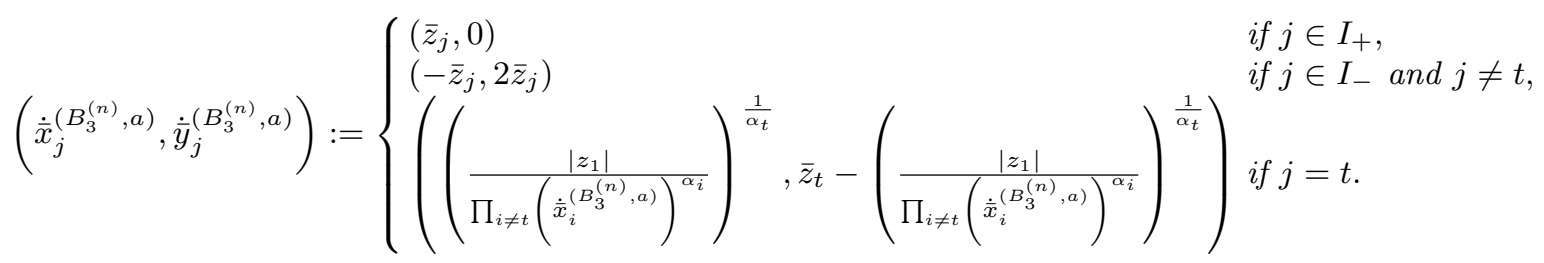

Similarly, $q$ is the smallest index in $I_{0}$ and $\dot{\bar{x}}^{\left(B_{3}^{(n)}, b\right)}, \dot{\bar{y}}^{\left(B_{3}^{(n)}, b\right)}$ are respectively defined as follows:

$$
\left(\dot{\bar{x}}_{j}^{\left(B_{3}^{(n)}, b\right)}, \dot{\bar{y}}_{j}^{\left(B_{3}^{(n)}, b\right)}\right):= \begin{cases}\left(\bar{z}_{j}, 0\right) & \text { if } j \in I_{+}, \\
\left(-\bar{z}_{j}, 2 \bar{z}_{j}\right) & \text { if } j \in I_{-}, \\
(1,-1) & \text { if } j \in I_{0} \text { and } j \neq q, \\
\left(\left(\frac{\left|z_{1}\right|}{\prod_{i \neq q}\left(\dot{\bar{x}}_{i}^{\left(B_{3}^{(n)}, b\right)}\right)^{\alpha_{i}}}\right)^{\frac{1}{\alpha_{q}}},-\left(\frac{\left|z_{1}\right|}{\prod_{i \neq q}\left(\dot{\bar{x}}_{i}^{\left(B_{3}^{(n)}, b\right)}\right)^{\alpha_{i}}}\right)^{\frac{1}{\alpha_{q}}}\right. & \begin{array}{l}
\text { if } j=q .
\end{array}\end{cases}
$$

(d) If $z \in B_{4}^{(n)}$, then

$$
z=1 \cdot\left[\begin{array}{c}
0 \\
\mathbf{1}^{(n)}-\mathbf{1}_{k}
\end{array}\right]+1 \cdot\left[\begin{array}{c}
0 \\
\mathbf{1}_{k}-\mathbf{1}^{(n)}
\end{array}\right]
$$

where $\mathbf{1}_{k}(k=1,2, \cdots, n)$ is the kth column of the identity matrix $I_{n}$. 
Theorem 6 For any given $z=\left(z_{1}, \bar{z}\right) \in \mathbb{R} \times \mathbb{R}^{n}$, its Type II decomposition with respect to $\mathcal{K}_{\alpha}^{(n)}$ is given by

(a) If $z \in B_{1}^{(n)}$, then

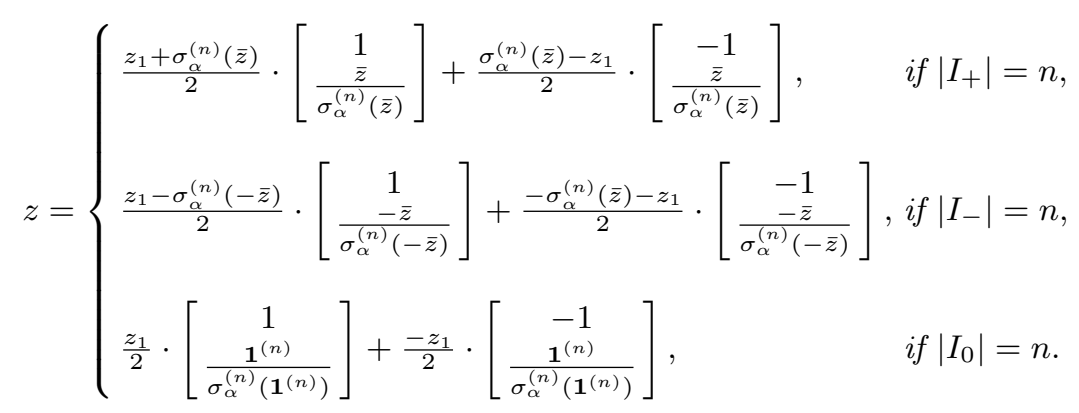

(b) If $z \in B_{2}^{(n)}$, then

$$
z=\left\{\begin{array}{l}
1 \cdot\left[\begin{array}{c}
z_{1} \\
\ddot{\bar{x}}^{\left(B_{2}^{(n)}, a\right)}
\end{array}\right]+(-1) \cdot\left[\begin{array}{c}
0 \\
\ddot{\bar{y}}^{\left(B_{2}^{(n)}, a\right)}
\end{array}\right], \text { if }\left|I_{-}\right|=0, \\
(-1) \cdot\left[\begin{array}{c}
-z_{1} \\
\ddot{\bar{x}}^{\left(B_{2}^{(n)}, b\right)}
\end{array}\right]+1 \cdot\left[\begin{array}{c}
0 \\
\ddot{\bar{y}}^{\left(B_{2}^{(n)}, b\right)}
\end{array}\right], \text { if }\left|I_{+}\right|=0,
\end{array}\right.
$$

where $k$ is the smallest index in $I_{0}$ and $\ddot{\bar{x}}_{2}^{\left(B_{2}^{(n)}, a\right)}, \ddot{\bar{y}}^{\left(B_{2}^{(n)}, a\right)}, \ddot{\bar{x}}_{2}^{\left(B^{(n)}, b\right)}, \ddot{\bar{y}}^{\left(B_{2}^{(n)}, b\right)}$ are respectively defined as follows:

$$
\begin{aligned}
& \left(\ddot{\bar{x}}_{j}^{\left(B_{2}^{(n)}, a\right)}, \ddot{\bar{y}}_{j}^{\left(B_{2}^{(n)}, a\right)}\right):= \begin{cases}\left(\bar{z}_{j}, 0\right) & \begin{array}{l}
(1,1) \\
\text { if } j \in I_{+},
\end{array} \\
\left(\left(\frac{\left|z_{1}\right|}{\prod_{i \neq k}\left(\ddot{\bar{x}}_{i}^{\left(B_{2}^{(n)}, a\right)}\right)^{\alpha_{i}}}\right)^{\frac{1}{\alpha_{k}}},\left(\frac{\left|z_{1}\right|}{\prod_{i \neq k}\left(\ddot{\bar{x}}_{i}^{\left(B_{2}^{(n)}, a\right)}\right)^{\alpha_{i}}}\right)^{\frac{1}{\alpha_{k}}}\right) & \text { if } j=k .\end{cases}
\end{aligned}
$$

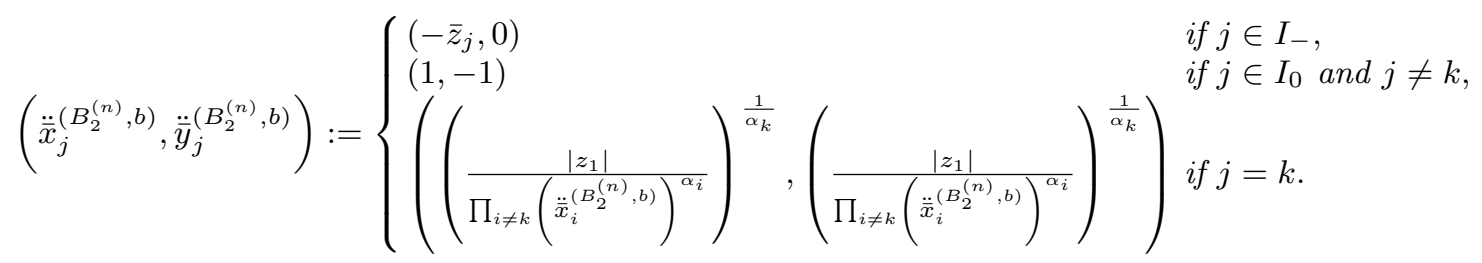

(c) If $z \in B_{3}^{(n)}$, then

$$
z=\left\{\begin{array}{l}
1 \cdot\left[\begin{array}{c}
z_{1} \\
\ddot{\bar{x}}^{\left(B_{3}^{(n)}, a\right)}
\end{array}\right]+(-1) \cdot\left[\begin{array}{c}
0 \\
\ddot{\bar{y}}^{\left(B_{3}^{(n)}, a\right)}
\end{array}\right], \text { if }\left|I_{0}\right|=0, \\
1 \cdot\left[\begin{array}{c}
z_{1} \\
\ddot{\bar{x}}^{\left(B_{3}^{(n)}, b\right)}
\end{array}\right]+(-1) \cdot\left[\begin{array}{c}
0 \\
\ddot{\bar{y}}^{\left(B_{3}^{(n)}, b\right)}
\end{array}\right], \text { if }\left|I_{0}\right| \neq 0,
\end{array}\right.
$$

where $t$ is the smallest index in $I_{-}$and $\ddot{\bar{x}}^{\left(B_{3}^{(n)}, a\right)}, \ddot{\bar{y}}^{\left(B_{3}^{(n)}, a\right)}$ are respectively defined as follows:

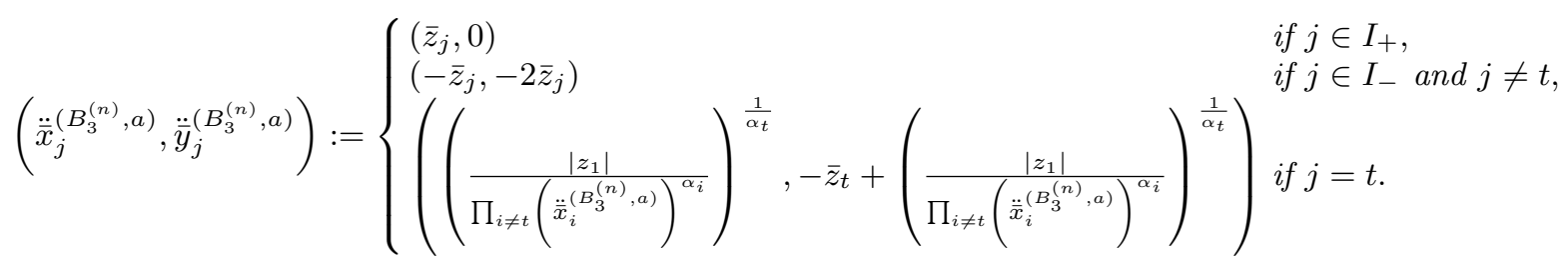


Similarly, $q$ is the smallest index in $I_{0}$ and $\ddot{\bar{x}}^{\left(B_{3}^{(n)}, b\right)}, \ddot{\bar{y}}^{\left(B_{3}^{(n)}, b\right)}$ are respectively defined as follows:

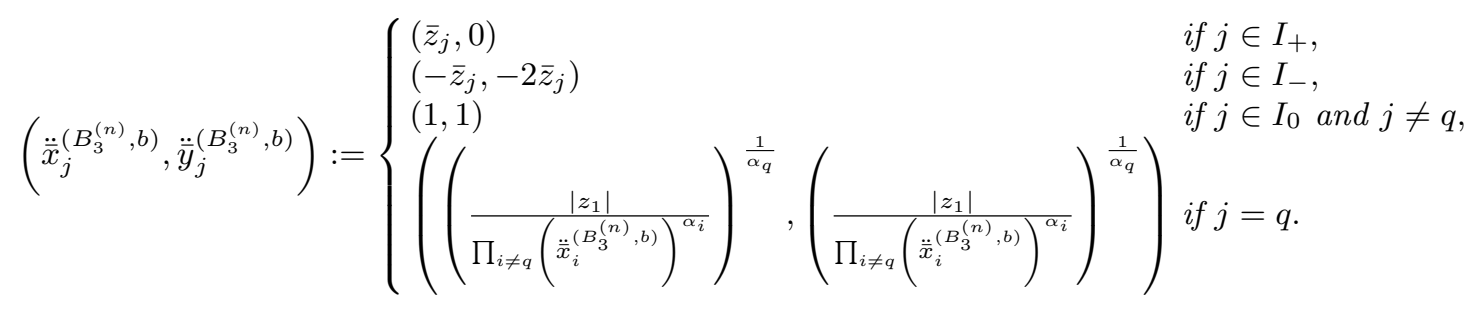

(d) If $z \in B_{4}^{(n)}$, then

$$
z=1 \cdot\left[\begin{array}{c}
0 \\
\mathbf{1}^{(n)}-\mathbf{1}_{k}
\end{array}\right]+(-1) \cdot\left[\begin{array}{c}
0 \\
\mathbf{1}^{(n)}-\mathbf{1}_{k}
\end{array}\right]
$$

\section{Concluding remarks}

In this paper, we propose two types of decomposition approaches for the power cone $\mathcal{K}_{\alpha}$ and the exponential cone $\mathcal{K}_{\text {exp }}$, which are the generators of many well-known nonsymmetric cones. In particular, the corresponding explicit decomposition formulas are established based on different classifications for the reference points with respect to the given cones and the decomposition types. In contrast to the setting of $\mathcal{K}_{\exp }$, the power cone $\mathcal{K}_{\alpha}$ seems to be more regular, because its two types of decompositions share the same space division. At the same time, we also define their conic functions, namely $f^{\text {power }}$ and $f^{\text {exp }}$ as Definition 1 and 2 . As a byproduct, we can extend the decomposition results of the power cone $\mathcal{K}_{\alpha}$ to its high-dimensional case $\mathcal{K}_{\alpha}^{(n)}$ by slight modifications.

Although the results are not quite complete due to the difficulty of handling nonsymmetric cones, they are very crucial to subsequent study towards nonsymmetric cone optimization. Further investigations are definitely desirable. We summarize and list out some future topics as below.

1. Exploring more structures and properties for the power cone and the exponential cone, such as their variational geometries including normal cones, tangent cones, second-order tangent sets, critical cone and "sigma" terms.

2. Similar to the second cone and its generalization circular cone, can the properties of continuity, strict continuity, Lipschitz continuity, directional differentiability, differentiability, continuous differentiability, and semismoothness be each inherited by $f^{\text {power }}$ and $f^{\text {exp }}$ from $f$ ?

3. Designing new algorithms for these nonsymmetric cones based on the non-interior-point framework, such as augmented Lagrangian method, proximal point method and their variants.

On the other hand, there are so many non-symmetric cones in real world. Can we figure out a way to clarify them? This is another important direction for our future study.

Acknowledgements The first author's work is supported by National Natural Science Foundation of China (Grant Number: 11601389). The third author's work is supported by Ministry of Science and Technology, Taiwan.

\section{Appendix}

\subsection{The concepts of $\alpha$-representable and extended $\alpha$-representable sets}

For a given convex set $\mathcal{K}$, it is $\alpha$-representable [4, Page 110] if there exist a finite integer $M$, scalars $\alpha_{i} \in[0,1]$, $i=1,2, \cdots, M$, vectors $c_{1}, c_{2}, \cdots, c_{M} \in \mathbb{R}^{3}$, matrices $A_{1}, A_{2}, \cdots, A_{M}$ with three columns and an appropriate 
number of rows, a matrix $A_{f}$ and a vector $c_{f}$ such that

$$
u \in \mathcal{K} \Leftrightarrow c_{i}-A_{i}^{T}\left[\begin{array}{l}
u \\
v
\end{array}\right] \in \mathcal{K}_{\alpha_{i}}(i=1,2, \cdots, M), A_{f}^{T}\left[\begin{array}{l}
u \\
v
\end{array}\right]=c_{f}
$$

for some artificial variables or modelling variables $v$. Similarly, the set $\mathcal{K}$ is extended $\alpha$-representable $[4$, Page $122]$ if there exist finite integers $M_{1}, M_{2}$, matrices $A_{\alpha}, A_{\exp }, A_{f}$ and vectors $c_{\alpha}, c_{\exp }, c_{f}$ of appropriate sizes such that

$$
u \in \mathcal{K} \Leftrightarrow c_{\alpha}-A_{\alpha}^{T}\left[\begin{array}{l}
u \\
v
\end{array}\right] \in \prod_{i=1}^{M_{1}} \mathcal{K}_{\alpha_{i}}, c_{\exp }-A_{\exp }^{T}\left[\begin{array}{l}
u \\
v
\end{array}\right] \in \prod_{i=1}^{M_{2}} \mathcal{K}_{\exp }, A_{f}^{T}\left[\begin{array}{l}
u \\
v
\end{array}\right]=c_{f}
$$

\subsection{Optimization models involving the power cone and the exponential cone}

Location problem $[4,19]$ : The generalized location problem is to find a point $x \in \mathbb{R}^{n}$ whose sum of weight distances from a given set of locations $L_{1}, \cdots, L_{m}$ is minimized, which has the following form

$$
\text { (P) } \min _{x \in \mathbb{R}^{n}} \sum_{i=1}^{m} w_{i}\left\|x-L_{i}\right\|_{p_{i}}
$$

where $\|\cdot\|_{p_{i}}\left(p_{i} \geq 1\right)$ denotes the $p_{i}$-norm defined on $\mathbb{R}^{n}$. If $p_{i}$ is equal to 2 , then the above problem reduces to the classical Weber-Point problem. Denote by $x:=\left(x_{1}, \cdots, x_{n}\right)^{T} \in \mathbb{R}^{n}$ and $a:=\left(a_{1}, \cdots, a_{n}\right)^{T} \in \mathbb{R}^{n}$, Problem $(P)$ can be rewritten as

$$
\begin{array}{ll}
\min _{x, a, y_{i}} & \sum_{i=1}^{m} w_{i} a_{i} \\
\text { s.t. } & \left(y_{i, j}, a_{i}, x_{j}-L_{i, j}\right) \in \mathcal{K}_{\frac{1}{p_{i}}}, i=1, \cdots, m, j=1, \cdots, n, \\
& \sum_{j=1}^{n} y_{i, j}=a_{i}, i=1, \cdots, m,
\end{array}
$$

where $L_{i, j}$ and $y_{i, j}$ stand for the $j$-th component of $L_{i} \in \mathbb{R}^{n}$ and $y_{i} \in \mathbb{R}^{n}$, respectively.

Geometric programming $[3,31,34]$ : Let $x:=\left(x_{1}, \cdots, x_{n}\right)^{T} \in \mathbb{R}^{n}$ be a vector with real positive components $x_{i}$. A real valued function $m$, of the form $m(x):=c \prod_{i=1}^{n} x_{i}^{\alpha_{i}}$, is called a monomial function, where $c>0$ and $\alpha_{i}$ are its coefficient and exponents, respectively. A sum of one or more monomials, i.e., a function that looks like $f(x):=\sum_{k=1}^{K} m_{k}(x)$, is called a posynomial function, where $m_{k}(x):=c_{k} \prod_{i=1}^{n} x_{i}^{\alpha_{i, k}}$. A geometric program is composed of a posynomial objective with posynomial inequality constraints and monomial equality constraints, which can be described as

$$
\begin{array}{ll}
\min _{x} & f_{0}(x) \\
\text { s.t. } & f_{s}(x) \leq 1, \quad s=1, \cdots, p, \\
& g_{t}(x)=1, \quad t=1, \cdots, q,
\end{array}
$$

where $f_{s}:=\sum_{k=1}^{K} c_{k, s} \prod_{i=1}^{n} x_{i}^{\alpha_{i, k, s}}, s \in\{0,1, \cdots, p\}$ and $g_{t}(x):=c_{t} \prod_{i=1}^{n} x_{i}^{\alpha_{i, t}}, t \in\{1, \cdots, q\}$. Using the following change of variables as $x_{i}:=\exp \left(u_{i}\right), c_{k, i}:=\exp \left(d_{k, i}\right), c_{t}:=\exp \left(d_{t}\right)$ and adding some additional variables, Problem (GP) can be rewritten as

$$
\begin{array}{ll}
\min _{u_{i}, w, \xi_{k, 0}, \eta_{k, s}} & w \\
\text { s.t. } & \left(d_{k, 0}+\sum_{i=1}^{n} u_{i} \cdot \alpha_{i, k, 0}, \xi_{k, 0}, 1\right) \in \mathcal{K}_{\exp }, \sum_{k=1}^{K} \xi_{k, 0}=w, \\
& \left(d_{k, s}+\sum_{i=1}^{n} u_{i} \cdot \alpha_{i, k, s}, \eta_{k, s}, 1\right) \in \mathcal{K}_{\exp }, \sum_{k=1}^{K} \eta_{k, s}=1, \quad s=1, \cdots, p, \\
& d_{t}+\sum_{i=1}^{n} u_{i} \cdot \alpha_{i, t}=0, \quad t=1, \cdots, q .
\end{array}
$$




\subsection{The decomposition with respect to the circular cone}

Consider the circular cone

$$
\mathcal{L}_{\theta}:=\left\{\left(x_{1}, \bar{x}\right) \in \mathbb{R} \times \mathbb{R}^{n-1} \mid x_{1} \tan \theta \geq\|\bar{x}\|\right\} .
$$

For any given $z=\left(z_{1}, \bar{z}\right) \in \mathbb{R} \times \mathbb{R}^{n-1}$, the projection mappings $\Pi_{\mathcal{L}_{\theta}}(z), \Pi_{\mathcal{L}_{\theta}^{\circ}}(z)$ are respectively given by

$$
\Pi_{\mathcal{L}_{\theta}}(z):=\left\{\begin{array}{l}
z, \text { if } z \in \mathcal{L}_{\theta}, \\
0, \text { if } z \in \mathcal{L}_{\theta}^{\circ}, \\
u, \text { otherwise },
\end{array} \quad \Pi_{\mathcal{L}_{\theta}^{\circ}}(z):=\left\{\begin{array}{l}
0, \text { if } z \in \mathcal{L}_{\theta}, \\
z, \text { if } z \in \mathcal{L}_{\theta}^{\circ}, \\
v, \text { otherwise }
\end{array}\right.\right.
$$

where

$$
u=\left[\begin{array}{c}
\frac{z_{1}+\left\|z_{2}\right\| \tan \theta}{1+\tan ^{2} \theta} \\
\left(\frac{z_{1}+\left\|z_{2}\right\| \tan \theta}{1+\tan ^{2} \theta} \tan \theta\right) \frac{z_{2}}{\left\|z_{2}\right\|}
\end{array}\right], \quad v=\left[\begin{array}{c}
\frac{z_{1}-\left\|z_{2}\right\| \cot \theta}{1+\cot ^{2} \theta} \\
\left(\frac{z_{1}-\left\|z_{2}\right\| \cot \theta}{1+\cot ^{2} \theta} \cot \theta\right) \frac{-z_{2}}{\left\|z_{2}\right\|}
\end{array}\right] .
$$

Combining these results with the Moreau decomposition theorem, the decomposition with respect to $\mathcal{L}_{\theta}$ is

$$
z=\tilde{\lambda}_{1}(z) \cdot \tilde{u}_{z}^{(1)}+\tilde{\lambda}_{2}(z) \cdot \tilde{u}_{z}^{(2)}
$$

where

$$
\begin{array}{cl}
\tilde{\lambda}_{1}(z):=z_{1}-\|\bar{z}\| \cot \theta, & \tilde{\lambda}_{2}(z):=z_{1}+\|\bar{z}\| \tan \theta \\
\tilde{u}_{z}^{(1)}:=\frac{1}{1+\cot ^{2} \theta}\left[\begin{array}{cc}
1 & 0 \\
0 & \cot \theta
\end{array}\right]\left[\begin{array}{c}
1 \\
-w
\end{array}\right], \tilde{u}_{z}^{(2)}:=\frac{1}{1+\tan ^{2} \theta}\left[\begin{array}{cc}
1 & 0 \\
0 & \tan \theta
\end{array}\right]\left[\begin{array}{c}
1 \\
w
\end{array}\right]
\end{array}
$$

with $w=\frac{\bar{z}}{\|\bar{z}\|}$ if $\bar{x} \neq 0$ and $w$ is any unit vector in $\mathbb{R}^{n-1}$ if $\bar{x}=0$. It is easy to see that

$$
\Pi_{\mathcal{L}_{\theta}}(z)=\max \left\{0, \tilde{\lambda}_{1}(z)\right\} \cdot \tilde{u}_{z}^{(1)}+\max \left\{0, \tilde{\lambda}_{2}(z)\right\} \cdot \tilde{u}_{z}^{(2)}
$$

More properties of the circular cone can be found in [45, Section 3].

\subsection{Proof of Lemma 1}

By definition, $\mathcal{K}_{\alpha}$ is closed, since the functions $\bar{x}_{1}^{\alpha_{1}} \bar{x}_{2}^{\alpha_{2}}$ and $\left|x_{1}\right|$ are continuous on $\mathbb{R}_{+}^{2}$ and $\mathbb{R}$, respectively. To proof that $\mathcal{K}_{\alpha}$ is a convex cone, we only need to verify that it is closed under the addition and the nonnegative multiplication. For any given $\left(x_{1}, \bar{x}\right) \in \mathcal{K}_{\alpha}$ and $\beta \geq 0$, one can obtain that

$$
\left(\beta \bar{x}_{1}\right)^{\alpha_{1}}\left(\beta \bar{x}_{2}\right)^{\alpha_{2}}=\beta \bar{x}_{1}^{\alpha_{1}} \bar{x}_{2}^{\alpha_{2}} \geq \beta\left|x_{1}\right|=\left|\beta x_{1}\right|, \beta \bar{x}_{1} \geq 0, \beta \bar{x}_{2} \geq 0
$$

where the first equation uses the fact $\alpha_{1}+\alpha_{2}=1$. Therefore, we have $\beta\left(x_{1}, \bar{x}\right) \in \mathcal{K}_{\alpha}$. For any given $\left(x_{1}, \bar{x}\right),\left(y_{1}, \bar{y}\right) \in \mathcal{K}_{\alpha}$, we know

$$
\begin{aligned}
& \left|x_{1}\right| \leq \bar{x}_{1}^{\alpha_{1}} \bar{x}_{2}^{\alpha_{2}}, \bar{x}_{1} \geq 0, \bar{x}_{2} \geq 0 \\
& \left|y_{1}\right| \leq \bar{y}_{1}^{\alpha_{1}} \bar{y}_{2}^{\alpha_{2}}, \bar{y}_{1} \geq 0, \bar{y}_{2} \geq 0 .
\end{aligned}
$$

It is easy to see that $\bar{x}_{1}+\bar{y}_{1} \geq 0, \bar{x}_{2}+\bar{y}_{2} \geq 0$ and $\left|x_{1}+y_{1}\right| \leq\left|x_{1}\right|+\left|y_{1}\right| \leq \bar{x}_{1}^{\alpha_{1}} \bar{x}_{2}^{\alpha_{2}}+\bar{y}_{1}^{\alpha_{1}} \bar{y}_{2}^{\alpha_{2}}$. In order to finish our proof, it suffices to show that

$$
\bar{x}_{1}^{\alpha_{1}} \bar{x}_{2}^{\alpha_{2}}+\bar{y}_{1}^{\alpha_{1}} \bar{y}_{2}^{\alpha_{2}} \leq\left(\bar{x}_{1}+\bar{y}_{1}\right)^{\alpha_{1}}\left(\bar{x}_{2}+\bar{y}_{2}\right)^{\alpha_{2}}, \forall\left(x_{1}, \bar{x}\right),\left(y_{1}, \bar{y}\right) \in \mathcal{K}_{\alpha}
$$

We divide it into the following two cases. Suppose that there exists an index $i \in\{1,2\}$ such that $\bar{x}_{i}=0$ or $\bar{y}_{i}=0$, it is trivial to show (52). Otherwise, we obtain $\bar{x}, \bar{y} \in \mathbb{R}_{++}^{2}$. Consider the function $f: \mathbb{R}_{++}^{2} \rightarrow \mathbb{R}$ :

$$
f(\bar{x})=\bar{x}_{1}^{\alpha_{1}} \bar{x}_{2}^{\alpha_{2}}
$$

where $\bar{x}:=\left(\bar{x}_{1}, \bar{x}_{2}\right)^{T} \in \mathbb{R}^{2}$ and $\bar{x}_{1}, \bar{x}_{2}>0$. By calculation, we obtain

$$
\nabla^{2} f(\bar{x})=\left[\begin{array}{cc}
\alpha_{1}\left(\alpha_{1}-1\right) \bar{x}_{1}^{\alpha_{1}-2} & 0 \\
0 & \alpha_{2}\left(\alpha_{2}-1\right) \bar{x}_{2}^{\alpha_{2}-2}
\end{array}\right]
$$


Since $\alpha_{i} \in(0,1)$ and $\bar{x}_{i}$ is strictly positive, the Hessian matrix $\nabla^{2} f(\bar{x})$ is negative definite, which shows that $f$ is concave defined on $\mathbb{R}_{++}^{2}$. Therefore, we have

$$
f\left(\frac{\bar{x}+\bar{y}}{2}\right) \geq \frac{1}{2}(f(\bar{x})+f(\bar{y}))
$$

which is equivalent to the above inequality (52).

\section{References}

1. Alizadeh,F., Goldfarb,D.: Second-order cone programming. Math. Program. 95(1), 3-51 (2003)

2. Andersen,E.D., Roos,C., Terlaky,T.: Notes on duality in second order and p-order cone optimization. Optimization $\mathbf{5 1}(4), 627-643(2002)$

3. Boyd,S., Kim,S.J., Vandenberghe,L, Hassibi,A.: A tutorial on geometric programming. Optim. Eng. \{bf 8$\}$, 67-127 (2007)

4. Chares, R.: Cones and interior-point algorithms for structured convex optimization involving powers and exponentials. PhD thesis, UCL-Universite Catholique de Louvain (2009)

5. Chua, C.B.: A $t$-algebraic approach to primal-dual interior-point algorithms. SIAM J. Optim., 20, 503-523 (2009).

6. Chang, Y.L., Yang, C.Y., Chen, J.S.: Smooth and nonsmooth analysis of vector-valued functions associated with circular cones. Nonlinear Anal. 85, 160-173 (2013)

7. Chen, J.S., Chen,X., Tseng,P.: Analysis of nonsmooth vector-valued functions associated with second-order cone. Math. Program. 101(1), 95-117 (2003)

8. Chen, J.S., Pan, S.H.: A entropy-like proximal algorithm and the exponential multiplier method for symmetric cone programming. Comput. Optim. Appl. 47(3), 477-499 (2010)

9. Chen, J.S., Tseng, P.: An unconstrained smooth minimization reformulation of second-order cone complementarity problem. Math. Program. 104(2-3), 293-327 (2005)

10. Chen, J.S.: SOC functions and their applications, Springer Optimization and Its Applications 143, Springer, Singapore (2019).

11. Ding, C., Sun, D.F., Toh, K.C.: An introduction to a class of matrix cone programming. Math. Program., 144, 141-179 (2014)

12. Dür, M.: Copositive programming-a survey, in Recent Advances in Optimization and its Applications in Engineering, edited by M. Diehl et al, Springer-Verlag, Berlin, (2010)

13. Faraut, U., Korányi, A.: Analysis on Symmetric Cones. University Press, Oxford (1994)

14. Fukushima, M., Luo, Z.Q., Tseng, P.: Smoothing functions for second-order-cone complementarity problems. SIAM J. Optim. 12(2), 436-460 (2001)

15. Glineur, F.: An extended conic formulation for geometric optimization. Found. Comput. Decision Sci. 25, 161-174 (2000)

16. Glineur, F.: Proving strong duality for geometric optimization using a conic formulation. Ann. Oper. Res. 105, 155-184 (2001)

17. Glineur, F., Terlaky, T.: Conic formulation for $l_{p}$-norm optimization. J. Optim. Theory Appl. 122, 285-307 (2004)

18. Güler O.: Hyperbolic polynomials and iterior point methods for convex programming. Math. Oper. Res. 22, 350-377 (1997)

19. Khanh Hien, L.: Differential properties of Euclidean projection onto power cone. Math. Meth. Oper. Res. 82(3), 265-284 (2015)

20. Bauschke, H.H., Güler, O., Lewis, A.S., Sendov, H.S.: Hyperbolic polynomials and convex analysis. Canad. J. Math. 53, 470-488 (2001)

21. Ito, M., Lourenco, B.F.: The $p$-cone in dimension $n \geq 3$ are not homogeneous when $p \neq 2$. Linear Algebra Appl. 533, 326-335 (2017)

22. Lobo, M.S., Vandenberghe, L., Boyd, S., Lebret, H.: Applications of second-order cone programming. Linear Algebra Appl. 284(1-3), 193-228 (1998)

23. Karimi, M., Tuncel, L.: Primal-dual interior-point methods for domain-driven formulations: algorithms. arXiv preprint arXiv:1804.06925.

24. Mordukhovich, B.S., Outrata, J.V., Ramiez C.H.: Second-order variational analysis in conic programming with applications to optimality and stability. SIAM J. Optim. 25(1), 76-101 (2015)

25. Moreau. J.J.: Décomposition orthogonale d'un espace hilbertien selon deux cônes mutuellement polaires. Comptes Rendus de l'Académie des Sciences 255, 238-240 (1962)

26. Miao, X.H., Lu, Y., Chen, J.S.: From symmetric cone optimization to nonsymmetric cone optimization: Spectral decomposition, nonsmooth analysis, and projections onto nonsymmetric cones. Pac. J. Optim. 14(3), 399-419 (2018)

27. Miao, X.H., Qi, N., Chen, J.S.: Projection formula and one type of spectral factorization associated with $p$-order cone. J. Nonlinear Convex Anal. 18(9), 1699-1705 (2017)

28. Nesterov, Y.: Towards non-symmetric conic optimization. Optim. Methods Softw. 27, $893-917$ (2012)

29. Pan, S.H., Chang, Y.L., Chen, J.S.: Stationary point conditions for the FB merit function associated with symmetric cones. Oper. Res. Lett. 38(5), 372-377 (2010)

30. Pan, S.H., Chen, J.S.: An R-linearly convergent nonmonotone derivative-free method for symmetric cone complementarity problems. Adv. Model. Optim. 13, 185-211 (2011)

31. Peres, Y., Pete G., and Somersille S.: Biased tug-of-war, the biased infinity Laplacian, and comparison with exponential cones, Calculus of Variations 38, 541-564 (2010)

32. Renegar, J.: Hyperbolic program and their derivative relaxations. Found. Comput. Math. 6, 59-79 (2006)

33. Schmieta, S.H., Alizadeh, F.: Extension of primal-dual interior point algorithms to symmetric cones. Math. Program. 96(3), 409-438 (2003)

34. Serrano, S.A.: Algorithms for unsymmetric cone optimization and an implementation for problems with the exponential cone. PhD thesis, Stanford University (2015)

35. Shapiro, A.: First and second order analysis of nonlinear semidefinite programs. Math. Program. 77(1), 301-320 (1997) 
36. Skajaa, A., Ye., Y.Y.: A homogeneous interior-point algorithm for nonsymmetric convex conic optimization. Math. Program. 150, 391-422 (2015)

37. Sun, D.F.: The strong second-order sufficient condition and constraint nondegeneracy in nonlinear semidefinite programming and their implications. Math. Oper. Res. 31(4), 761-776 (2006)

38. Sun, D.F., Sun, J.: Löwner's operator and spectral functions in Euclidean Jordan algebras. Math. Oper. Res. 33(2), 421-445 (2008)

39. Sun, D.F., Sun, J., Zhang, L.W.: The rate of convergence of the augmented Lagrangian method for nonlinear semidefinite programming. Math. Program. 114(2), 349-391 (2008)

40. Tseng, P.: Merit functions for semi-definite complementarity problems. Math. Program. 83(2), 159-185 (1998)

41. Vandenberghe, L., Boyd, S.: Semidefinite programming. SIAM Review 38(1), 49-95 (1996)

42. Vinberg, E.B.: The theory of homogeneous convex cones. Trans. Moscow Math. Soc. 12 340-403 (1963) (English Translation)

43. Wolkowicz, H., Saigal, R., Vandenberghe, L., Eds.: Handbook of Semidefinite Programming, Kluwer Academic, Boston (2000)

44. Xue, G.L., Ye, Y.Y.: An efficient algorithm for minimizing a sum of $p$-norm. SIAM J. Optim. 10(2), 551-579 (2000)

45. Zhou, J.C., Chen, J.S.: Properties of circular cone and spectral factorization associated with circular cone. J. Nonlinear Convex Anal. 14(4), 807-816 (2013) 